\title{
Effects of eight-quark interactions on the hadronic vacuum and mass spectra of light mesons
}

\author{
A.A. Osipov ${ }^{*}, 1$, B. Hiller, A.H. Blin, J. da Providência \\ Centro de Física Teórica, Departamento de Física da Universidade de Coimbra, 3004-516 Coimbra, Portugal
}

Received 4 August 2006; accepted 22 August 2006

Available online 6 October 2006

\begin{abstract}
The combined effective low energy QCD Lagrangians of Nambu-Jona-Lasinio (NJL) and 't Hooft are supplemented with eight-quark interactions. This work is a follow-up of recent findings, namely (i) the six quark flavour determinant 't Hooft term destabilizes the NJL vacuum, (ii) the addition of a chiral invariant eight-fermion contact term renders the ground state of the theory globally stable; (iii) stability constrains the values of coupling constants of the model, meaning that even in the presence of eight-quark forces the system can be unstable in a certain parameter region. In the present work we study a phenomenological output of eight-quark interactions considering the mass spectra of pseudoscalar and scalar mesons. Mixing angles are obtained and their equivalence to the two angle approach is derived. We show that the masses of pseudoscalars are almost neutral to the eight-quark forces. The only marked effect of the second order in the SU(3) breaking is found in the $\eta-\eta^{\prime}$ system. The scalars are more sensitive to the eight-quark interactions. A strong repulsion between the singlet and octet members is the reason for the obtained low mass of the $\sigma$ state within the model considered.

(c) 2006 Elsevier Inc. All rights reserved.
\end{abstract}

PACS: $12.39 . \mathrm{Fe} ; 11.30 . \mathrm{Rd} ; 11.30 . \mathrm{Qc}$

\footnotetext{
${ }^{*}$ Corresponding author. Fax: +74962166666 .

E-mail addresses: osipov@nusun.jinr.ru (A.A. Osipov), brigitte@teor.fis.uc.pt (B. Hiller), alex@teor.fis.uc.pt (A.H. Blin), providencia@teor.fis.uc.pt (J. da Providência).

${ }^{1}$ On leave from Joint Institute for Nuclear Research, Laboratory of Nuclear Problems, 141980 Dubna, Moscow Region, Russia.
} 


\section{Introduction}

The fundamental fields of QCD, quarks and gluons, are unobservable dynamical variables. Instead, at low energies, one observes hadrons. The most direct way to study their properties is the method of effective Lagrangians written in terms of the matter fields describing mesons or baryons. Such theories, under some circumstances, can be developed to the advanced level of an effective field theory. A well-known example is chiral perturbation theory [1], where the Lagrangian of light pseudoscalar mesons is both a derivative and light current quark mass expansion around the asymmetric ground state which is assumed to be stable. This stability is a phenomenological fact; the underlying theory must explain it.

It is commonly accepted that the eight approximate Goldstone bosons $\pi, K$, and $\eta$ are a signal for the spontaneous breakdown of the chiral symmetry of QCD, which is realized in the ideal world of massless $u, d$, and $s$ quarks. It is not excluded that effective four-quark interactions of the Nambu and Jona-Lasinio (NJL) type [2] are responsible for the formation of a stable chiral asymmetric vacuum giving a crude insight into the structure of the ground state of QCD [3].

One might ask if higher order quark interactions are of importance. For instance, on lines suggested by an instanton-gas model, it can be argued [4] that there exists an infinite set of multi-quark terms in the effective quark Lagrangian starting from the NJL fourquark interactions. The famous 't Hooft determinantal interaction [5] automatically appears if one keeps only the zero mode contribution in the mode expansion of the effective Lagrangian. This $2 N_{f}$ multi-quark term ( $N_{f}$ being the number of quark flavours) manifestly violates the $U_{A}(1)$ axial symmetry of the QCD Lagrangian, offering a way out of the $U_{A}(1)$ problem.

Let us recall the case with the lightest flavour singlet pseudoscalar $\eta^{\prime}$, which was for a while a deep theoretical problem, known as the $U_{A}(1)$ puzzle. The general solution [6] has shown that the $\eta^{\prime}$, being a quark-antiquark state, is strongly connected to the gluon world and that the $U_{A}(1)$ axial anomaly is the reason for the $\eta^{\prime}-\pi, K$, and $\eta$ splitting observed in nature. These conclusions are based only on the Ward identities and the $1 / N_{\mathrm{c}}$ expansion of QCD (where $N_{\mathrm{c}}$ is the number of colours).

The same question has been also studied in the framework of an effective Lagrangian which includes the meson fields and the topological charge density $Q(x)$ [7]. After the elimination of the field $Q(x)$ by means of its classical equation of motion, one obtains an effective mesonic Lagrangian. It has been shown by Rosenzweig, Schechter, and Trahern [8] that the 't Hooft type determinantal interaction, written in terms of mesonic fields, appears as the first term in the expansion which results from eliminating $Q(x)$. From the Lagrangian of the model one realizes again that there are no valid theoretical objections against the idea that the 't Hooft interaction and higher order multiquark terms are actually present in the QCD vacuum. Part of these interactions have been utilized in [9].

Thus, it is tempting to consider the intuitive picture that describes the QCD vacuum with basis on a series of multi-quark interactions reflecting several tractable features of QCD, which include aspects of chiral symmetry and of the $1 / N_{\mathrm{c}}$ expansion. The bosonization of quark degrees of freedom leads then to the desirable effective Lagrangian with matter fields and a stable chiral asymmetric vacuum. 
This idea is not new. The NJL type model with the $U_{A}(1)$ axial symmetry breaking by the 't Hooft determinant (in the following we will use the abbreviation NJLH for this model) has been studied in the mean field approximation [10-16] for a long time. Numerous phenomenological applications show that the results of such an approach meet expectations. Nevertheless in this picture, there is an apparent problem: the mean field potential is unbounded from below, and the 't Hooft term is the direct source of such an instability (see, for instance, Eq. (3.16) in [15]). A consistent approach requires obviously a stable hadronic vacuum in which the pions would live forever in the ideal world with only strong interactions.

The functional integral bosonization of the model exposes new shortcomings: the system of stationary phase equations used to estimate the generating functional of the theory $Z$, has several real solutions [17] which contribute independently, i.e., $Z=Z_{1}+Z_{2}+\ldots+Z_{n}$, where $n$ is the total number of such real solutions. Since only one of them (let us assume $Z_{1}$, for definiteness) leads, at leading order, to the mean field potential, $V_{\mathrm{MF}}$, the semiclassical potential $V$, corresponding to $Z$, differs from $V_{\mathrm{MF}}$. It has been shown in [17] that $V$ is also unbounded from below. Thus, we must accept that the NJLH model suffers from a ground state problem.

Recently it has been argued [18] that eight-quark interactions, added to the NJLH Lagrangian, might resolve the problem. Indeed, the mean field potential of the modified theory, $\mathcal{V}_{\mathrm{MF}}$, has a globally stable minimum. The just mentioned controversy concerning the results obtained by the mean field method and the functional integral approach is also removed: one can prove that $\mathcal{V}=\mathcal{V}_{\mathrm{MF}}$, i.e., the number of admissible real solutions to the stationary phase equations can be constrained to one due to eight-quark terms.

There is a natural question. If the eight-quark forces are so important for the formation of the ground state, what are the other phenomenological consequences of such interactions?

In this paper we consider the main characteristics of light pseudoscalar mesons $\left(J^{\mathrm{PC}}=0^{-+}\right)$: their masses and weak decay constants. After that we switch to scalars, calculating masses of the $J^{\mathrm{PC}}=0^{++}$quark-antiquark nonet. The structure of scalars is a subject of many studies nowadays. The question is so complicated that it would be too naive to think that eight-quark forces are a panacea for the mass spectrum problem. Our aim is only to demonstrate the tendency. Once we understand what is changed by the new interactions considered in the description of the meson properties within the model, we can clarify the role of eight-quark forces for low-energy QCD.

To study this matter one should choose an appropriate approximation. The bosonization of six- and eight-quark interactions cannot be done exactly. We will use the stationary phase method to replace the multi-quark vertices by purely mesonic ones and by Yukawa type interactions of quarks with mesons. This is a standard approach [11,19-21]. The subsequent integration over quarks is a straightforward calculation, because one deals here with a Gaussian integral. To obtain the effective mesonic Lagrangian and extract masses, we shall expand the real part of the quark determinant in a heat kernel series [22,23]. The techniques which are particularly well suited to the present task have been developed in [24].

The outline of the paper is as follows: after introducing the multi-quark Lagrangian in Section 2.1, the stability conditions of the vacuum are discussed in Section 2.2. In Sections 2.3-2.5 we use bosonization and heat kernel methods to transform the multi-quark into a mesonic Lagrangian, and extract the relevant contributions to the 
mass spectra and gap-equations. Section 3 is dedicated to the analysis of the pseudoscalar observables and includes a detailed discussion of decay constants with particular emphasis on the relation of our one angle approach to the two mixing angle analysis. Explicit formulae for masses and mixing angles are obtained in Section 3.4. In Section 4 are presented the characteristics of scalars, numerical results are given in Section 5 and conclusions in Section 6. Two appendices contain, respectively, the detailed derivation of the uniqueness of the solutions of the stationary phase equations and of the solution of the equations which yield the matricial coefficients relevant for meson mass terms.

\section{The model}

\subsection{The multi-quark Lagrangian}

We discuss the system of light quarks $u, d, s\left(N_{f}=3\right)$ with multi-fermion interactions described by the Lagrangian

$$
\mathcal{L}_{\text {eff }}=\bar{q}\left(\mathrm{i} \gamma^{\mu} \partial_{\mu}-m\right) q+\mathcal{L}_{4 q}+\mathcal{L}_{6 q}+\mathcal{L}_{8 q}+\cdots .
$$

Quark fields $q$ have colour $\left(N_{\mathrm{c}}=3\right)$ and flavour indices which are suppressed. We suppose that four-, six-, and eight-quark interactions $\mathcal{L}_{4 q}, \mathcal{L}_{6 q}, \mathcal{L}_{8 q}$ are effectively local. Likewise, they are constructed from local quark bilinears, like the scalar $S_{a}=\bar{q} \lambda_{a} q$, or the pseudoscalar $P_{a}=\bar{q} \mathbf{i} \gamma_{5} \lambda_{a} q$ "currents". Such bilinears have the appropriate quantum numbers to describe mesons. This approximation corresponds to the task considered: we want to obtain, after bosonization, the tree level effective meson Lagrangian, with local vertices and local meson fields, and relate the coupling constants and masses of such a Lagrangian with the parameters of the quark model. Meson physics in the large $N_{\mathrm{c}}$ limit is described by a local Lagrangian of this type [25].

The global chiral $\mathrm{SU}(3)_{L} \times \mathrm{SU}(3)_{R}$ symmetry of the Lagrangian (1) at $m=0$ is spontaneously broken to the SU(3) group, showing the dynamical instability of the fully symmetric solutions of the theory. In addition, the current quark mass $m$, being a diagonal matrix in flavour space with elements $\operatorname{diag}\left(m_{u}, m_{d}, m_{s}\right)$, explicitly breaks this symmetry down, retaining only the reduced $\mathrm{SU}(2)_{I} \times U(1)_{Y}$ symmetries of isospin and hypercharge conservation, if $m_{u}=m_{d} \neq m_{s}$.

The leading order (in $N_{\mathrm{c}}$ counting) Lagrangian of light mesons and the corresponding underlying quark Lagrangian must inherit the $U(3)_{L} \times U(3)_{R}$ chiral symmetry of massless three-flavour QCD. In particular, it was argued [26] that in the large $N_{\mathrm{c}}$ limit of QCD with three massless quarks the pattern of spontaneous chiral symmetry breakdown is uniquely fixed: the chiral $U(3)_{L} \times U(3)_{R}$ group, under some highly plausible assumptions, necessarily breaks down to the diagonal $U(3)$. In accordance with these expectations the shortrange attractive $U(3)_{L} \times U(3)_{R}$ symmetric NJL-type interaction

$$
\mathcal{L}_{4 q}=\frac{G}{2}\left[\left(\bar{q} \lambda_{a} q\right)^{2}+\left(\bar{q} \mathrm{i} \gamma_{5} \lambda_{a} q\right)^{2}\right]
$$

can be used to specify the corresponding part of the effective quark Lagrangian in channels with quantum numbers $J^{P}=0^{+}, 0^{-}$[27]. The matrices acting in flavour space, $\lambda_{a}$, $a=0,1, \ldots, 8$, are normalized such that $\operatorname{tr}\left(\lambda_{a} \lambda_{b}\right)=2 \delta_{a b}$. Here $\lambda_{0}=\sqrt{\frac{2}{3}} 1$, and $\lambda_{k}$, 
$k=1,2, \ldots, 8$ are the standard SU(3) Gell-Mann matrices. It is well-known that such four-quark interactions lead (for some values of the model parameters) to the formation of a quark condensate, which is invariant under the vector subgroup $U(3)$ and thus breaks chiral invariance of the ground state in accordance with the requirements of three-flavour QCD.

The 't Hooft determinantal interaction is described by the Lagrangian

$$
\mathcal{L}_{6 q}=\kappa\left(\operatorname{det} \bar{q} P_{L} q+\operatorname{det} \bar{q} P_{R} q\right),
$$

where the matrices $P_{L, R}=\left(1 \mp \gamma_{5}\right) / 2$ are chiral projectors and the determinant is over flavour indices. This interaction breaks explicitly the axial $U_{A}(1)$ symmetry, lifting the degeneracy of $\eta$ and $\eta^{\prime}$ meson masses (in the chiral limit), and violates Zweig's rule [28] due to flavour mixing. It affects also the scalar singlet and octet states pushing down the mass of the SU(3) singlet.

The large $N_{\mathrm{c}}$ behaviour of the model is reflected in the dimensionfull coupling constants, $[G]=M^{-2},[\kappa]=M^{-5}$, which count as $G \sim 1 / N_{\mathrm{c}}, \kappa \sim 1 / N_{\mathrm{c}}^{N_{f}}$. As a result the NJL interaction (2) dominates over $\mathcal{L}_{6 q}$ at large $N_{\mathrm{c}}$, as one would expect, because Zweig's rule is exact at $N_{\mathrm{c}}=\infty$. These couplings have opposite signs: $G>0, \kappa<0$.

The eight-quark Lagrangian which describes the spin zero interactions contains two terms: $\mathcal{L}_{8 q}=\mathcal{L}_{8 q}^{(1)}+\mathcal{L}_{8 q}^{(2)}[18]$, where

$$
\begin{aligned}
\mathcal{L}_{8 q}^{(1)} & =8 g_{1}\left[\left(\bar{q}_{i} P_{R} q_{m}\right)\left(\bar{q}_{m} P_{L} q_{i}\right)\right]^{2}=\frac{g_{1}}{32}[\operatorname{tr}(S-\mathrm{i} P)(S+\mathrm{i} P)]^{2} \\
& =\frac{g_{1}}{8}\left(S_{a}^{2}+P_{a}^{2}\right)^{2}, \\
\mathcal{L}_{8 q}^{(2)} & =16 g_{2}\left[\left(\bar{q}_{i} P_{R} q_{m}\right)\left(\bar{q}_{m} P_{L} q_{j}\right)\left(\bar{q}_{j} P_{R} q_{k}\right)\left(\bar{q}_{k} P_{L} q_{i}\right)\right] \\
& =\frac{g_{2}}{16} \operatorname{tr}[(S-\mathrm{i} P)(S+\mathrm{i} P)(S-\mathrm{i} P)(S+\mathrm{i} P)] \\
& =\frac{g_{2}}{16} \operatorname{tr}\left(S^{4}+P^{4}+4 P^{2} S^{2}-2 P S P S\right) \\
& =\frac{g_{2}}{8}\left[d_{a b e} d_{c d e}\left(S_{a} S_{b} S_{c} S_{d}+P_{a} P_{b} P_{c} P_{d}+2 S_{a} S_{b} P_{c} P_{d}\right)+4 f_{a c e} f_{b d e} S_{a} S_{b} P_{c} P_{d}\right] .
\end{aligned}
$$

Here the trace is taken over flavour indices $i, j=1,2,3$; the matrices $S, P$ are given by $S_{i j}=S_{a}\left(\lambda_{a}\right)_{i j}=2 \bar{q}_{j} q_{i}, P_{i j}=P_{a}\left(\lambda_{a}\right)_{i j}=2 \bar{q}_{j}\left(i \gamma_{5}\right) q_{i}$. The $f_{a b c}$ are the well-known totally antisymmetric structure constants: $\left[\lambda_{a}, \lambda_{b}\right]=2 \mathrm{i} f_{a b c} \lambda_{c}$. The $d_{a b c}$ are totally symmetric quantities: $\left\{\lambda_{a}, \lambda_{b}\right\}=2 d_{a b c} \lambda_{c} . \mathcal{L}_{8 q}$ is a $U(3)_{L} \times U(3)_{R}$ symmetric interaction with OZI-violating effects in $\mathcal{L}_{8 q}^{(1)}$.

The eight-quark interactions $\mathcal{L}_{8 q}$ are the lowest order terms in number of quark fields which stabilize the vacuum state of the model. We restrict our consideration to theses terms, because in the long wavelength limit (or in the case when the multi-quark correlators create a hierarchy) the higher dimensional operators are suppressed.

Since the coupling constants $G, \kappa, g_{1}$, and $g_{2}$ are dimensionful, the model is not renormalizable. We use the cutoff $\Lambda$ to make quark loops finite. The regularization procedure (Pauli-Villars) is standard and can be found, for instance, in our paper [29], where the regularization function is introduced to define the coincidence limit of the Schwinger-DeWitt representation for the real part of the quark-loop effective action. This method is used for our calculations of mass spectra in the following. 


\subsection{Stability conditions for the vacuum}

The eight-quark forces stabilize the vacuum [18]. To clarify the meaning of this statement, consider, for the sake of simplicity, the effective potential $V(M)$ which one obtains as a result of bosonization of these multi-quark vertices in the chirally symmetric limit $(m=0)$ and in the one-quark-loop approximation

$$
V(M)=\frac{h^{2}}{16}\left(12 G+\kappa h+\frac{27}{2} \lambda h^{2}\right)-\frac{3 N_{\mathrm{c}}}{16 \pi^{2}}\left[M^{2} J_{0}\left(M^{2}\right)+\Lambda^{4} \ln \left(1+\frac{M^{2}}{\Lambda^{2}}\right)\right],
$$

with $\Lambda$ being an ultraviolet cutoff in the quark one-loop diagrams, and

$$
J_{0}\left(M^{2}\right)=\Lambda^{2}-M^{2} \ln \left(1+\frac{\Lambda^{2}}{M^{2}}\right) .
$$

The dependence on the variable $h$ is defined by the stationary phase equation

$$
M+G h+\frac{\kappa}{16} h^{2}+\frac{3}{4} \lambda h^{3}=0, \quad \lambda \equiv g_{1}+\frac{2}{3} g_{2}
$$

as a function of the model parameters and the argument $M$.

We start the discussion of the effective potential with the standard case of four-quark interactions, where the curvature of the potential at the origin and the sign of the coupling $G$ of the interaction fully determine the existence of a globally stable system, which can occur either in the Wigner-Weyl or in the phase of spontaneously broken chiral symmetry. Often the word "instability" is used in connection with the transition from this symmetric to the spontaneously broken vacuum at a critical value of $G \Lambda^{2}$. This is not what is meant when we say that the vacuum is unstable. As we hope will be clear after the discussion presented in the remaining of this section, the instability we refer to is an essential pathology of the vacuum, present in the model with combined four- and six-quark interactions: we show that it is crucial for the stability of the vacuum that the stationary phase equation (8) possesses only one single real root when higher order multi-quark interactions are present. We argue that the enlarged system with six-quark interactions fails in this respect and that eight-quark interactions are necessary to stabilize the vacuum. Fig. 1 will illustrate the various stages of the discussion.

In a world without six- and eight-quark interactions, $\kappa, g_{1}, g_{2}=0$, one obtains from Eq. (8) that $h=-M / G$ and, as a result, the potential $V(M)$ has the form of a double well, if $V^{\prime \prime}(0)<0$, see Fig. 1d, i.e., if $\tau$, the following combination of model parameters:

$$
\tau=\frac{N_{\mathrm{c}} G \Lambda^{2}}{2 \pi^{2}}>1
$$

This inequality expresses the fact that chiral symmetry is spontaneously broken, ${ }^{2}$ producing in the massless case, $(m=0)$, the degeneracy of a nonet of Goldstone bosons, and showing the presence of the $U_{A}(1)$ problem. This vacuum state is globally stable, because at large values of $|M|$ another inequality is fulfilled

$$
V(M) \sim \frac{3 G}{4} h^{2}(M)=\frac{3 M^{2}}{4 G}>0 \quad(M \rightarrow \pm \infty)
$$

\footnotetext{
${ }^{2}$ The Wigner-Weyl phase appears for $0<\tau<1$, i.e., $V^{\prime \prime}(0)>0$, see Fig. 1a.
} 

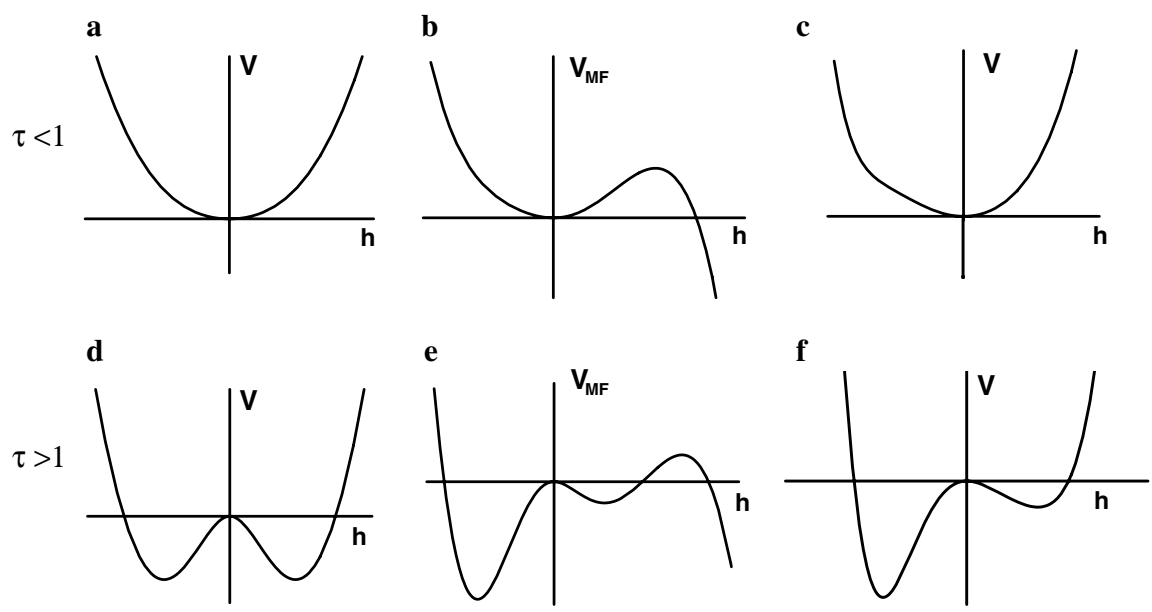

Fig. 1. The effective potential $V$ in the SU(3) chiral limit, as function of the quark condensate related variable $h$. Upper and lower panels are classified by the value of $\tau=\frac{N_{\mathrm{c}} G \Lambda^{2}}{2 \pi^{2}}$, related to the curvature of the effective potential at the origin. Each panel shows the typical form of the potential when one adds successively to the four-quark (see a, $d$ on the left), the six- (middle figures b, e) and eight-quark interactions (figures c, f on the right). The metastable cases (b) and (e) are obtained in the mean field approximation $V_{\mathrm{MF}}$ (the stationary phase approach leads to an unstable vacuum, without any local minimum, [17]).

at $G>0$. In this particular case the stability of the ground state $(G>0)$ is already guaranteed by Eq. (9).

It is worth noting that higher order multi-quark interactions will not change condition (9) as long as Eq. (8) has only one real solution. The reason for this is very simple. If this equation has only one real solution, it is valid to expect that $h(M)=-M / G+\mathcal{O}\left(M^{2}\right)$. Since $2 n$-quark vertices contribute to $V(M)$ as $h^{n} \sim M^{n}$, the value of $V^{\prime \prime}(0)$ is entirely determined by terms of the second power in $M$, i.e., by the four-quark interaction $(n=2)$ only. Therefore it is tempting to describe the general situation (when higher order multi-quark interactions are included) by the same inequality (9), since it will dictate the behavior of the effective potential in the neighbourhood of the origin in full agreement with the leading order result; this is illustrated in Fig. 1, the upper panel for $\tau<1$, the lower one for $\tau>1$. For that one must find however a way to reduce the number of real roots of the corresponding stationary phase equation to one.

What is wrong with several roots? Let us consider the system which includes four- and six-quark interactions $G, \kappa \neq 0, g_{1}, g_{2}=0$. In this case the quadratic Eq. (8) has two solutions, both being real for $M \geqslant 4 G^{2} / \kappa$. It follows then that the stationary phase method leads us to the gap equation which contains the sum of these solutions

$$
h^{(1)}+h^{(2)}+\frac{N_{\mathrm{c}} M}{\pi^{2}} J_{0}\left(M^{2}\right)=0
$$

The sum does not depend on $M$, because $h^{(1)}+h^{(2)}=-16 G / \kappa$, and this gap equation misses the trivial solution $M=0$, corresponding to the chiral symmetric vacuum. One sees that a simple addition of the 't Hooft interaction to the four-quark Lagrangian affects so violently 
the trivial solution and as a matter of fact the whole effective potential, which gets unstable, ${ }^{3}$ that, apparently, we must get rid of the problem which appears as soon as the stationary phase equation has more than one real solution. ${ }^{4}$

Since a quadratic equation never has only one real root, we are pushed to increase the order of the equation by including eight-quark interactions. One obtains in this way the cubic equation (8). This equation has only one real solution $h(M)$, which changes smoothly in the open interval $-\infty<h<\infty$ being an isomorphic and monotonic function of $M$, when one restricts the choice of parameters to

$$
G>\frac{1}{\lambda}\left(\frac{\kappa}{24}\right)^{2}, \quad \lambda>0
$$

For this case the constituent quark mass $M$ fulfills the gap equation

$$
h(M)+\frac{N_{\mathrm{c}} M}{2 \pi^{2}} J_{0}\left(M^{2}\right)=0
$$

related with the potential $V(M)$ (see Eq. (6)) which is bounded from below (see Figs. 1c and f).

Eq. (12) replace the previous requirement $G>0$ (see Eq. (10)). They must be fulfilled to guarantee the global stability of the system. The first inequality is new and plays for the enlarged system the role of Eq. (10): in the case of four-quark interactions only, the linear stationary phase equation had automatically only one real root, here the values of couplings must be fixed correspondingly to ensure the existence of only one real root. The second inequality is a direct analogue of $G>0$.

Eq. (9) is still relevant to the case and is responsible for the behaviour of $V(M)$ in the neighbourhood of zero, as mentioned before. In Figs. 1c and $\mathrm{f}$ the stabilizing effect due to the addition of eight-quark interactions is shown. Note that they change radically the potential only at values of $h>-8 G / \kappa$, as compared to the cases $1 \mathrm{~b}$ and $1 \mathrm{e}$, calculated in the mean field approximation (see also footnote 4), affecting little the other branch of the potential, where $h \sim h^{(1)}$. In particular the value of $h$ where the global minimum of both potentials occurs in the spontaneously broken phase, is negative. Since at the quark one-loop order $h$ is proportional to the quark condensate [29], one is inclined to believe that by fixing the model parameters through it, this will finally lead to similar numerical values for all observables which depend in a stringent way on the value of the condensate. For those observables, the calculations in the metastable mean-field approximation and in the globally stable case considered with inclusion of the eight-quark interactions will not differ much.

Another interesting aspect of this simple analysis of the SU(3) limit of the effective potential is the possibility of existence of multiple vacua, illustrated in Fig. 2. For $\tau<1$, i.e., in a region where the four-quark interactions alone lead to the symmetric WignerWeyl phase, the inclusion of the 't Hooft six-quark interactions can induce spontaneous symmetry breaking for some critical values of the coupling parameter $\kappa$. This new vacuum

\footnotetext{
3 This point has been considered in detail in [17].

${ }^{4}$ Here we would like to stress that Figs. $1 \mathrm{~b}$ and e, related with the addition of six-quark interactions, are obtained within the mean field approach, which leads to the effective potential (6) taken at $\lambda=0$ and considered as a function of $h$ [15]. We identify it with $V_{\mathrm{MF}}(h)$. The dependence $M(h)$ is given by Eq. (8). This is a one-to-one mapping $h \rightarrow M$, where $h$ ranges along the interval $-\infty<h<\infty$. The local maximum at positive $h=-8 G / \kappa$ on both figures corresponds to the point where the regular (at $\kappa \rightarrow 0$ ) solution $h^{(1)}$ changes to the singular $h^{(2)}$. $V_{\mathrm{MF}}(h)$ is unbounded from below, as $h^{(2)} \rightarrow \infty$.
} 


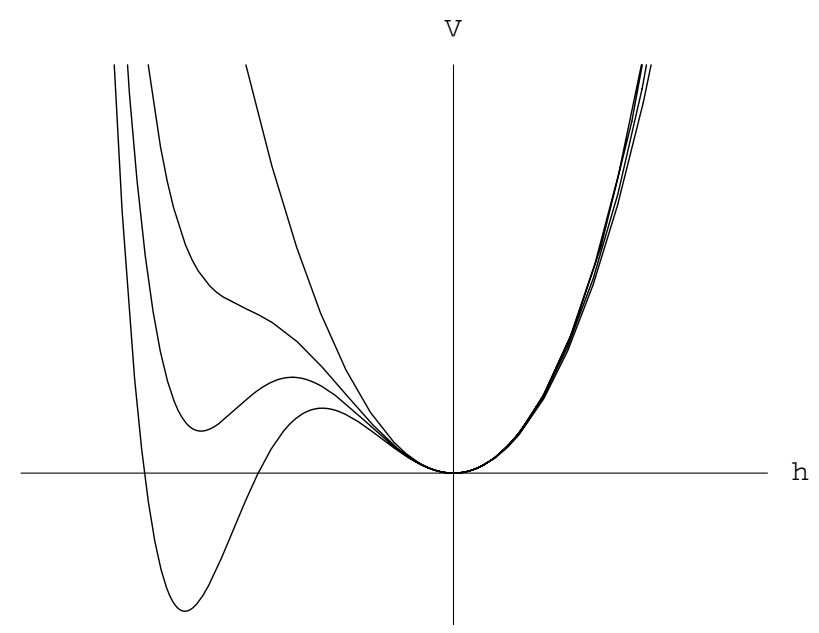

Fig. 2. A closer view on the effective potential $V$ of Fig. 1c. Depending on the strength of the six-quark coupling $\kappa$, with remaining parameters $G, \lambda, \Lambda$ fixed, the symmetric Wigner-Weyl phase of the four-quark potential (Fig. 1a) may coexist with the spontaneously broken phase induced by the presence of the 't Hooft term. This double vacuum exists within the global stability conditions (12).

can coexist with the trivial vacuum. This has been discussed previously in [30], now we are able to confirm its existence within the general stability conditions imposed for the vacuum, given by the inequalities in (12). In the presence of non-vanishing values for the current quark mass, the minimum at the origin shifts towards the physical region of negative $h$. The phenomenon of multiple vacua has been addressed in several other approaches to the description of the QCD vacuum [31-33].

The most general case is obtained if the $\mathrm{SU}(3)_{L} \times \mathrm{SU}(3)_{R}$ chiral symmetry of the quark Lagrangian is broken down explicitly by the non-zero values of current quark masses $m$. Then the inequalities (12) must be replaced by the following ones [18]

$$
g_{1}>0, \quad g_{1}+3 g_{2}>0, \quad G>\frac{1}{g_{1}}\left(\frac{\kappa}{16}\right)^{2} .
$$

The last constraint can be used to make a large $N_{c}$ estimate for $g_{1}$. Indeed, we know that $G$ scales as $1 / N_{\mathrm{c}}, \kappa \sim 1 / N_{\mathrm{c}}^{3}$ and, therefore, conclude from the above inequality that $g_{1}$ cannot scale as $1 / N_{\mathrm{c}}^{6}$ or smaller. On the other hand, this eight-quark interaction is an additional (to the 't Hooft determinant) source of OZI-violating effects and thus it cannot be stronger than the 't Hooft interactions, i.e., $g_{1} \sim 1 / N_{\mathrm{c}}^{4}$ or less. These reasonings show that $1 / N_{\mathrm{c}}^{5} \leqslant g_{1} \leqslant 1 / N_{\mathrm{c}}^{4}$. We would expect $g_{2}$ to be in the same interval, although inequalities (14) are not appropriate to prove that.

\subsection{Semi-bosonized Lagrangian}

The multi-quark Lagrangian (1) can be presented in the bilinear form with respect to the quark fields. The details can be found in [11,17]. Without using any approximations one obtains the following vacuum-to-vacuum amplitude of the theory 


$$
\begin{aligned}
Z= & \int \mathcal{D} q \mathcal{D} \bar{q} \prod_{a} \mathcal{D} \sigma_{a} \prod_{a} \mathcal{D} \phi_{a} \exp \left(\mathrm{i} \int \mathrm{d}^{4} x \mathcal{L}_{q}(\bar{q}, q, \sigma, \phi)\right) \\
& \times \int_{-\infty}^{+\infty} \prod_{a} \mathcal{D} s_{a} \prod_{a} \mathcal{D} p_{a} \exp \left(\mathrm{i} \int \mathrm{d}^{4} x \mathcal{L}_{r}(\sigma, \phi, \Delta ; s, p)\right)
\end{aligned}
$$

where

$$
\begin{aligned}
\mathcal{L}_{q}= & \bar{q}\left(\mathrm{i} \gamma^{\mu} \partial_{\mu}-M-\sigma-\mathrm{i} \gamma_{5} \phi\right) q, \\
\mathcal{L}_{r}= & s_{a}\left(\sigma_{a}+\Delta_{a}\right)+p_{a} \phi_{a}+\frac{G}{2}\left(s_{a}^{2}+p_{a}^{2}\right) \\
& +\frac{\kappa}{32} A_{a b c} s_{a}\left(s_{b} s_{c}-3 p_{b} p_{c}\right)+\frac{g_{1}}{8}\left(s_{a}^{2}+p_{a}^{2}\right)^{2} \\
& +\frac{g_{2}}{8}\left[d_{a b e} d_{c d e}\left(s_{a} s_{b} s_{c} s_{d}+2 s_{a} s_{b} p_{c} p_{d}+p_{a} p_{b} p_{c} p_{d}\right)\right. \\
& \left.+4 f_{\text {ace }} f_{b d e} s_{a} s_{b} p_{c} p_{d}\right] .
\end{aligned}
$$

The bosonic fields $\sigma=\sigma_{a} \lambda_{a}$ and $\phi=\phi_{a} \lambda_{a}$ are the composite scalar and pseudoscalar nonets which will be identified later with the corresponding physical states. The auxiliary fields $s_{a}$ and $p_{a}$ must be integrated out from the effective mesonic Lagrangian $\mathcal{L}_{r}$. The quarks obtain their constituent masses $M=M_{a} \lambda_{a}=\operatorname{diag}\left(M_{u}, M_{d}, M_{s}\right)$ due to dynamical chiral symmetry breaking in the physical vacuum state, $\Delta_{a}=M_{a}-m_{a}$. The totally symmetric constants $A_{a b c}$ are related to the flavour determinant, and equal to

$$
A_{a b c}=\frac{1}{3 !} \epsilon_{i j k} \epsilon_{m n l}\left(\lambda_{a}\right)_{i m}\left(\lambda_{b}\right)_{j n}\left(\lambda_{c}\right)_{k l} \text {. }
$$

Some useful relations for $A_{a b c}$ can be found in [30].

\subsection{Stationary phase approximation for $Z$}

The functional integrals over auxiliary variables $s_{a}, p_{a}$ in Eq. (15) can be calculated approximately within the stationary phase method. For that one should first find all real stationary phase trajectories $s_{a}^{\text {st }}=s_{a}(\sigma, \phi), p_{a}^{\text {st }}=p_{a}(\sigma, \phi)$ given by the system of equations

$$
\frac{\partial \mathcal{L}_{r}}{\partial s_{a}}=0, \quad \frac{\partial \mathcal{L}_{r}}{\partial p_{a}}=0 .
$$

We seek these solutions in form of expansions in the external mesonic fields $\sigma_{a}, \phi_{a}$

$$
\begin{aligned}
& s_{a}^{\mathrm{st}}=h_{a}+h_{a b}^{(1)} \sigma_{b}+h_{a b c}^{(1)} \sigma_{b} \sigma_{c}+h_{a b c}^{(2)} \phi_{b} \phi_{c}+\cdots \\
& p_{a}^{\mathrm{st}}=h_{a b}^{(2)} \phi_{b}+h_{a b c}^{(3)} \phi_{b} \sigma_{c}+\cdots
\end{aligned}
$$

The coefficients $h_{a \ldots}^{(i)}$ depend on the coupling constants $G, \kappa, g_{1}, g_{2}$, and quark masses $\Delta_{a}$. The higher index coefficients $h_{a \ldots}^{(i)}$ are recurrently expressed in terms of the lower ones. The one-index coefficients $h_{a}$ are the solutions of the following system of cubic equations

$$
\Delta_{a}+G h_{a}+\frac{3 \kappa}{32} A_{a b c} h_{b} h_{c}+\frac{g_{1}}{2} h_{a} h_{b}^{2}+\frac{g_{2}}{2} d_{a b e} d_{c d e} h_{b} h_{c} h_{d}=0
$$

Thus, the problem is reduced to a finite set of algebraic equations which span the nonet space of $U(3)$. These equations may be considered as an example of response equations, 
previously studied in [34] for the purpose of gaining some understanding about the possible origins of strong SU(3) breaking. The response $h_{a}$ is fully determined by the couplings $G, \kappa, g_{1}, g_{2}$, and the mean field $\Delta_{a}$, which plays the role of an external force. In accordance with the pattern of explicit symmetry breaking the mean field has only three non-zero components, with indices $a=0,3,8$.

In order to solve Eq. (21), one has to find whether there exists an intersection of a number of hypersurfaces. The important question of completeness of the system (21) is examined in Appendix A. This yields $h_{a}=0$ for $a=1,2,4,5,6,7$. Thus, the system reduces to three coupled equations to determine $h_{0}, h_{3}$, and $h_{8}$. This task has been solved in [18]. At this stage, as we have already discussed, one has to find conditions (see Eq. (14)) which ensure the one-to-one mapping $\Delta_{a} \leftrightarrow h_{a}$.

The next two equations following from (19) determine the coefficients $h_{a r}^{(1)}, h_{a r}^{(2)}$ in (20)

$$
\begin{aligned}
& \left\{\left(G+\frac{g_{1}}{2} h_{b}^{2}\right) \delta_{a r}+\frac{3 \kappa}{16} A_{a b r} h_{b}+g_{1} h_{a} h_{r}+\frac{g_{2}}{2}\left(2 d_{a b e} d_{r c e}+d_{c b e} d_{r a e}\right) h_{c} h_{b}\right\} h_{r s}^{(1)}=-\delta_{a s}, \\
& \left\{\left(G+\frac{g_{1}}{2} h_{b}^{2}\right) \delta_{a r}-\frac{3 \kappa}{16} A_{a b r} h_{b}+\frac{g_{2}}{2}\left(2 f_{\text {abe }} f_{r c e}+d_{c b e} d_{r a e}\right) h_{c} h_{b}\right\} h_{r s}^{(2)}=-\delta_{a s} .
\end{aligned}
$$

Corresponding solutions are given in Appendix B.

This procedure can be easily extended. Equating to zero the factor at any independent field combination in (19), one obtains an equation which determines one of the coefficients in (20).

On the other hand, these equations are useful if one wants to find the projection of the Lagrangian $\mathcal{L}_{r}$ on the stationary phase trajectory (20). For that one should rewrite them in a more convenient form, using that

$$
\begin{aligned}
& \left(h^{(1)}\right)_{a r}^{-1} h_{r}=G h_{a}+2 \Delta_{a}-\frac{g_{1}}{2} h_{a} h_{b}^{2}-\frac{g_{2}}{2} d_{a b e} d_{r c e} h_{b} h_{c} h_{r}, \\
& -\left(h^{(2)}\right)_{a r}^{-1} h_{r}=3 G h_{a}+2 \Delta_{a}+3 \frac{g_{1}}{2} h_{a} h_{b}^{2}+3 \frac{g_{2}}{2} d_{a b e} d_{c d e} h_{b} h_{c} h_{d}+g_{2} f_{a c e} f_{r d e} h_{c} h_{d} h_{r} .
\end{aligned}
$$

In particular, solutions of Eqs. (21)-(23) define the first three coupling constants of such Lagrangian, i.e., one can show that

$$
\mathcal{L}_{r} \rightarrow \mathcal{L}_{\mathrm{st}}=h_{a} \sigma_{a}+\frac{1}{2} h_{a b}^{(1)} \sigma_{a} \sigma_{b}+\frac{1}{2} h_{a b}^{(2)} \phi_{a} \phi_{b}+\mathcal{O}\left(\text { field }^{3}\right)
$$

Since the system of equations (19) can be solved, we are able to obtain the semi-classical asymptotics of the functional integral over $s_{a}$ and $p_{a}$ in $Z$. If parameters $G, \kappa, g_{1}$, and $g_{2}$ belong to a range where the system has a unique real solution, the calculations are straightforward. In particular, one has the following result which is valid at lowest order of the stationary phase approximation

$$
\begin{aligned}
& \int_{-\infty}^{+\infty} \prod_{a} \mathcal{D} s_{a} \prod_{a} \mathcal{D} p_{a} \exp \left(\mathrm{i} \int \mathrm{d}^{4} x \mathcal{L}_{r}(\sigma, \phi, \Delta ; s, p)\right) \\
& \sim \exp \left(\mathrm{i} \int \mathrm{d}^{4} x \mathcal{L}_{\mathrm{st}}(\sigma, \phi)\right) \quad(\hbar \rightarrow 0) .
\end{aligned}
$$




\subsection{Integrating quark fields in $Z$}

To obtain the effective Lagrangian of the model we should integrate out quark fields from Eq. (15). This is a well studied part of the calculations and we restrict ourselves to several general remarks here.

The one-quark-loop effective action can be computed in euclidean spacetime, the chiral invariant part of the result, $W_{q}[\sigma, \phi]$, is given by the modulus of the quark determinant

$$
W_{q}[\sigma, \phi]=\ln \left|\operatorname{det} D_{\mathrm{E}}\right|,
$$

where $D_{\mathrm{E}}$ stands for the Dirac operator in euclidean spacetime, namely $D_{\mathrm{E}}=\mathrm{i} \gamma_{\mu} \partial_{\mu}-M-\sigma-\mathrm{i} \gamma_{5} \phi$. The quark determinant is a complicated nonlocal functional which can be approximated in the low-energy regime by Schwinger-DeWitt asymptotic expansion $[22,23]$. The presence of a noncommutative (with respect to the bosonic fields $\sigma$ and $\phi$ ) mass matrix $M$ requires a more delicate treatment of this term in comparison with the standard approach, where $M$ is supposed to commutative with the fields. The corresponding technique has been recently developed [24] and applied to the case considered here in [29]. We refer to these papers for necessary details (see Section 3 in [29]), although we present the result, because we need it in the following.

The heat kernel expansion used is

$$
W_{q}[\sigma, \phi]=-\int \frac{\mathrm{d}^{4} x_{E}}{32 \pi^{2}} \sum_{i=1}^{\infty} I_{i-1} \operatorname{tr}\left(b_{i}\right),
$$

where coefficients $b_{i}$ for the case with isospin symmetry are

$$
b_{1}=-Y, \quad b_{2}=\frac{Y^{2}}{2}+\frac{\Delta_{u s}}{\sqrt{3}} \lambda_{8} Y, \quad \ldots
$$

Our following result is based on these two terms of the series. It is the lowest order approximation, because the usual kinetic term of the collective fields is contained in $b_{2}$, and we truncate the series exactly after this term. The part of $b_{2}$ with $\Delta_{u s}=M_{u}^{2}-M_{s}^{2}$ is absent from the standard Seeley-DeWitt coefficient $a_{2}$. This is one of the new features of the approach, which follows from the noncommutativity of the constituent quark mass matrix $M$.

The trace in Eq. (29) should be taken over colour, flavour and four-spinors indices. In Eq. (30) $Y$ is used for

$$
Y=\mathrm{i} \gamma_{\mu}\left(\partial_{\mu} \sigma+\mathrm{i} \gamma_{5} \partial_{\mu} \phi\right)+\sigma^{2}+[M, \sigma]+\phi^{2}+\mathrm{i} \gamma_{5}[\sigma+M, \phi] .
$$

The factors $I_{i}$ are given by the average

$$
I_{i}=\frac{1}{3}\left[2 J_{i}\left(M_{u}^{2}\right)+J_{i}\left(M_{s}^{2}\right)\right]
$$

and represent one-quark-loop integrals. In the considered approximation we need only to know $J_{0}\left(M^{2}\right)$ (see Eq. (7)) and

$$
J_{1}\left(M^{2}\right)=\ln \left(1+\frac{\Lambda^{2}}{M^{2}}\right)-\frac{\Lambda^{2}}{\Lambda^{2}+M^{2}} .
$$

We are using here the proper time regularization scheme. 
Thus, the integration over quark fields yields the second part of the effective Lagrangian (the first part is given by Eq. (26))

$$
\mathcal{L}_{q} \rightarrow \mathcal{L}_{h k}=\mathcal{L}_{\text {tad }}+\mathcal{L}_{\text {kin }}+\mathcal{L}_{m}+\mathcal{L}_{\text {int }}
$$

The tadpole term, $\mathcal{L}_{\text {tad }}$, is

$$
\mathcal{L}_{\mathrm{tad}}=\frac{N_{\mathrm{c}}}{12 \pi^{2}}\left[M_{u}\left(3 I_{0}-\Delta_{u s} I_{1}\right)\left(\sigma_{u}+\sigma_{d}\right)+M_{s}\left(3 I_{0}+2 \Delta_{u s} I_{1}\right) \sigma_{s}\right] .
$$

The kinetic term, $\mathcal{L}_{\text {kin }}$, after continuation to the Minkowski spacetime, requires a redefinition of meson fields to obtain the standard factor in front, i.e.,

$$
\mathcal{L}_{\text {kin }}=\frac{N_{\mathrm{c}} I_{1}}{16 \pi^{2}} \operatorname{tr}\left[\left(\partial_{\mu} \sigma\right)^{2}+\left(\partial_{\mu} \phi\right)^{2}\right]=\frac{1}{4} \operatorname{tr}\left[\left(\partial_{\mu} \sigma_{R}\right)^{2}+\left(\partial_{\mu} \phi_{R}\right)^{2}\right],
$$

where

$$
\sigma^{a}=g \sigma_{R}^{a}, \quad \phi^{a}=g \phi_{R}^{a}, \quad g^{2}=\frac{4 \pi^{2}}{N_{\mathrm{c}} I_{1}} .
$$

The contribution to the mass Lagrangian is given by

$$
\begin{aligned}
\mathcal{L}_{m}= & \frac{N_{\mathrm{c}} I_{0}}{4 \pi^{2}}\left(\sigma_{a}^{2}+\phi_{a}^{2}\right)-\frac{N_{\mathrm{c}} I_{1}}{12 \pi^{2}}\left\{\Delta_{u s}\left[2 \sqrt{2}\left(3 \sigma_{0} \sigma_{8}+\phi_{0} \phi_{8}\right)-\phi_{8}^{2}+\phi_{i}^{2}\right]\right. \\
& +2\left(2 M_{u}^{2}+M_{s}^{2}\right) \sigma_{0}^{2}+\left(M_{u}^{2}+5 M_{s}^{2}\right) \sigma_{8}^{2}+\left(7 M_{u}^{2}-M_{s}^{2}\right) \sigma_{i}^{2}+\left(M_{u}+M_{s}\right)\left(M_{u}+2 M_{s}\right) \sigma_{f}^{2} \\
& \left.+\left(M_{s}-M_{u}\right)\left(2 M_{s}-M_{u}\right) \phi_{f}^{2}\right\},
\end{aligned}
$$

where we assume that the indices $i$ and $f$ range over the subsets $i=1,2,3$ and $f=4,5,6,7$ of the set $a=0,1, \ldots, 8$. Thus we have

$$
\begin{aligned}
& \phi_{i}^{2}=2 \pi^{+} \pi^{-}+\left(\pi^{0}\right)^{2}, \quad \phi_{f}^{2}=2\left(K^{+} K^{-}+\bar{K}^{0} K^{0}\right), \\
& \sigma_{i}^{2}=2 a_{0}^{+} a_{0}^{-}+\left(a_{0}^{0}\right)^{2}, \quad \sigma_{f}^{2}=2\left(K_{0}^{*+} K_{0}^{*-}+\bar{K}_{0}^{* 0} K_{0}^{* 0}\right) .
\end{aligned}
$$

\section{Pseudoscalars: masses, mixings, and all that}

\subsection{Symmetry and currents}

One can simply obtain the conserved (or partially conserved) currents of the local theory by using the variational method of Gell-Mann and Lévy [35]. For instance, the infinitesimal local chiral transformations of the quark fields in $\mathcal{L}_{\text {eff }}$ (see Eq. (1)) are

$$
\delta q=\mathrm{i}\left(\alpha+\gamma_{5} \beta\right) q, \quad \delta \bar{q}=-\mathrm{i} \bar{q}\left(\alpha-\gamma_{5} \beta\right),
$$

where the small parameters $\alpha=\alpha_{a} \frac{\lambda_{a}}{2}$ and $\beta=\beta_{a} \frac{\lambda_{a}}{2}$ are Hermitian flavour matrices. Then, according to the Gell-Mann-Lévy formula, one obtains the standard vector $V_{\mu}^{a}$ and axial-vector $A_{\mu}^{a}$ nonet quark currents

$$
V_{\mu}^{a}=-\frac{\delta \mathcal{L}_{\mathrm{eff}}}{\delta\left(\partial \alpha_{a}\right)}=\bar{q} \gamma_{\mu} \frac{\lambda_{a}}{2} q, \quad A_{\mu}^{a}=-\frac{\delta \mathcal{L}_{\mathrm{eff}}}{\delta\left(\partial \beta_{a}\right)}=\bar{q} \gamma_{\mu} \gamma_{5} \frac{\lambda_{a}}{2} q,
$$

and their divergences 


$$
\begin{aligned}
\partial^{\mu} V_{\mu}^{a} & =-\frac{\delta \mathcal{L}_{\text {eff }}}{\delta \alpha_{a}}=\frac{\mathrm{i}}{2} \bar{q}\left[m, \lambda_{a}\right] q, \\
\partial^{\mu} A_{\mu}^{a} & =-\frac{\delta \mathcal{L}_{\text {eff }}}{\delta \beta_{a}}=\frac{\mathrm{i}}{2} \bar{q} \gamma_{5}\left\{m, \lambda_{a}\right\} q+\mathrm{i} \delta_{a 0} \sqrt{6} \kappa\left(\operatorname{det} \bar{q} P_{L} q-\operatorname{det} \bar{q} P_{R} q\right) .
\end{aligned}
$$

Transformations (40) induce the correlated change in the flavour space of collective fields

$$
\delta \sigma_{R}=\mathrm{i}\left[\alpha, \sigma_{R}+M g^{-1}\right]+\left\{\beta, \phi_{R}\right\}, \quad \delta \phi_{R}=\mathrm{i}\left[\alpha, \phi_{R}\right]-\left\{\beta, \sigma_{R}+M g^{-1}\right\} .
$$

The quark Lagrangian, $\mathcal{L}_{\text {eff }}$, is approximated by the effective bosonized Lagrangian $\mathcal{L}_{\text {bos }}$

$$
\mathcal{L}_{\text {eff }} \rightarrow \mathcal{L}_{\text {bos }}=\mathcal{L}_{\text {st }}+\mathcal{L}_{h k}+\cdots,
$$

where dots correspond to all omitted terms due to the approximations made. Therefore, one can obtain the currents again, but now they will be written in terms of meson fields. Indeed, one has

$$
\begin{aligned}
& \mathcal{A}_{\mu}^{a}=\frac{1}{4} \operatorname{tr}\left[\left(\left\{\sigma_{R}+M g^{-1}, \partial_{\mu} \phi_{R}\right\}-\left\{\partial_{\mu} \sigma_{R}, \phi_{R}\right\}\right) \lambda_{a}\right]+\mathcal{O}\left(b_{3}\right), \\
& \mathcal{V}_{\mu}^{a}=-\frac{\mathrm{i}}{4} \operatorname{tr}\left[\left(\left[\sigma_{R}+M g^{-1}, \partial_{\mu} \sigma_{R}\right]+\left[\phi_{R}, \partial_{\mu} \phi_{R}\right]\right) \lambda_{a}\right]+\mathcal{O}\left(b_{3}\right) .
\end{aligned}
$$

These currents obviously depend on the order where the heat kernel series is truncated, because the coefficient $b_{2}$ and higher ones contain derivatives. The symbol $\mathcal{O}\left(b_{3}\right)$ shows that currents (46) and (47) have been obtained from the Lagrangian $\mathcal{L}_{h k}$ based on two terms of the asymptotic series (29), namely $b_{1}$ and $b_{2}$.

\subsection{Decay constants of pseudoscalars}

Let us calculate matrix elements of axial-vector currents

$$
\left\langle 0\left|\mathcal{A}_{\mu}^{a}(0)\right| \phi_{R}^{b}(p)\right\rangle=\mathrm{i} f^{a b} p_{\mu} .
$$

Using Eq. (46) one derives

$$
\begin{aligned}
& f^{00}=\frac{2 M_{u}+M_{s}}{3 g}, \quad f^{11}=f^{22}=f^{33}=\frac{M_{u}}{g}, \quad f^{44}=f^{55}=f^{66}=f^{77}=\frac{M_{u}+M_{s}}{2 g}, \\
& f^{88}=\frac{M_{u}+2 M_{s}}{3 g}, \quad f^{08}=f^{80}=\sqrt{2} \frac{M_{u}-M_{s}}{3 g} .
\end{aligned}
$$

It is not difficult to relate these abstract values with the experimentally measured decay constants of physical pseudoscalar states $P(x)$. For instance, the weak decay constants of the pion $\left(f_{\pi}\right)$ and kaon $\left(f_{K}\right)$ are defined by the corresponding isotopic components of the axial current $\mathcal{A}_{\mu}^{1+\mathrm{i} 2}$ and $\mathcal{A}_{\mu}^{4+\mathrm{i} 5}$, i.e.,

$$
\left\langle 0\left|\mathcal{A}_{\mu}^{1+\mathrm{i} 2}(0)\right| \pi(p)\right\rangle=\mathrm{i} \sqrt{2} f_{\pi} p_{\mu}, \quad\left\langle 0\left|\mathcal{A}_{\mu}^{4+\mathrm{i} 5}(0)\right| K(p)\right\rangle=\mathrm{i} \sqrt{2} f_{K} p_{\mu},
$$

and, therefore, one finds ${ }^{5}$

$$
f_{\pi}=\frac{M_{u}}{g}, \quad f_{K}=\frac{M_{s}+M_{u}}{2 g} .
$$

\footnotetext{
${ }^{5}$ We use the normalizations $f_{\pi}=92.42 \pm 0.26 \mathrm{MeV}$, and $f_{K}=113.00 \pm 1.03 \mathrm{MeV}$ [36].
} 
These are the Goldberger-Treiman relations at the quark level.

The decay constants in the $\eta-\eta^{\prime}$ system are defined

$$
\left\langle 0\left|\mathcal{A}_{\mu}^{a}(0)\right| P(p)\right\rangle=\mathrm{i} f_{P}^{a} p_{\mu}, \quad(a=0,8),
$$

where $P=\eta, \eta^{\prime}$. Each of the two mesons has both, singlet and octet components

$$
\left(\begin{array}{c}
\phi_{R}^{0} \\
\phi_{R}^{8}
\end{array}\right)=\left(\begin{array}{cc}
\cos \theta_{p} & -\sin \theta_{p} \\
\sin \theta_{p} & \cos \theta_{p}
\end{array}\right)\left(\begin{array}{l}
\eta^{\prime} \\
\eta
\end{array}\right)
$$

This orthogonal rotation diagonalizes the kinetic and mass terms in the meson effective Lagrangian. The mixing angle $\theta_{p}$ will be calculated in Section 3.4. Consequently, from Eq. (52) one obtains the $2 \times 2$ matrix $\left\{f_{P}^{a}\right\}$

$$
\left\{f_{P}^{a}\right\}=\left(\begin{array}{cc}
f_{\eta}^{8} & f_{\eta}^{0} \\
f_{\eta^{\prime}}^{8} & f_{\eta^{\prime}}^{0}
\end{array}\right)=\left(\begin{array}{cc}
\cos \theta_{p} & -\sin \theta_{p} \\
\sin \theta_{p} & \cos \theta_{p}
\end{array}\right)\left(\begin{array}{ll}
f^{88} & f^{08} \\
f^{80} & f^{00}
\end{array}\right) .
$$

This matrix depends on four independent parameters $g, M_{u}, M_{s}$, and $\theta_{p}$. An alternative parametrization has been considered in $[37,38]$, where two constants $f_{0}, f_{8}$ and two angles $\vartheta_{0}, \vartheta_{8}$ specify the matrix $f_{P}^{a}$.

$$
\left\{f_{P}^{a}\right\}=\left(\begin{array}{cc}
f_{8} \cos \vartheta_{8} & -f_{0} \sin \vartheta_{0} \\
f_{8} \sin \vartheta_{8} & f_{0} \cos \vartheta_{0}
\end{array}\right)
$$

There is a straightforward correspondence between the parametrization of Kaiser and Leutwyler and the model predictions. Indeed, one finds

$$
\begin{aligned}
& \left(f_{8}\right)^{2}=\left(f_{\eta}^{8}\right)^{2}+\left(f_{\eta^{\prime}}^{8}\right)^{2}=\frac{1}{3 g^{2}}\left(M_{u}^{2}+2 M_{s}^{2}\right), \\
& \left(f_{0}\right)^{2}=\left(f_{\eta}^{0}\right)^{2}+\left(f_{\eta^{\prime}}^{0}\right)^{2}=\frac{1}{3 g^{2}}\left(2 M_{u}^{2}+M_{s}^{2}\right) .
\end{aligned}
$$

The formulae for the relations between mixing angles are given in the end of this section.

As one would expect, the model predictions agree well with the general requirements of chiral symmetry following from chiral perturbation theory (ChPT), although the results differ already at lowest order. For instance, we have

$$
\left(f_{8}\right)^{2}=\frac{4 f_{K}^{2}-f_{\pi}^{2}}{3}+\frac{\left(M_{s}-M_{u}\right)^{2}}{3 g^{2}} .
$$

One can show that the second term on the r.h.s. is of order $\left(m_{s}-m_{u}\right)^{2}$ and therefore must be omitted at lowest order of ChPT. The rest of this formula is a well-known low-energy relation which is valid in standard ChPT.

The mixing angles $\vartheta_{8}, \vartheta_{0}$ are small and $\vartheta_{8} \neq \vartheta_{0}$. We have for their difference

$$
\begin{aligned}
f_{8} f_{0} \sin \left(\vartheta_{8}-\vartheta_{0}\right) & =f_{\eta}^{8} f_{\eta}^{0}+f_{\eta^{\prime}}^{8} f_{\eta^{\prime}}^{0}=-\frac{\sqrt{2}}{3 g^{2}}\left(M_{s}^{2}-M_{u}^{2}\right) \\
& =-\frac{2 \sqrt{2}}{3}\left(f_{K}^{2}-f_{\pi}^{2}\right)-\frac{\sqrt{2}}{6 g^{2}}\left(M_{s}-M_{u}\right)^{2} .
\end{aligned}
$$

Thus, $\vartheta_{8} \neq \vartheta_{0}$ due to the SU(3) flavour symmetry breaking effect. Again, this result agrees with the ChPT formula, if one notes that the last term is a higher order contribution. 
The singlet decay constant $f_{0}$ is equal to

$$
\left(f_{0}\right)^{2}=\frac{2 f_{K}^{2}+f_{\pi}^{2}}{3}+\frac{f_{\pi}^{2}}{6}\left(\frac{M_{s}}{M_{u}}-1\right)^{2} .
$$

In the $\eta^{\prime}$-extended version of ChPT there is the OZI-rule violating term in the effective Lagrangian, which contributes as $f_{\pi}^{2} \Lambda_{1}$ to the r.h.s. of Eq. (60). We have instead the term $\left(M_{s} / M_{u}-1\right)^{2} / 6 \sim \Lambda_{1}$. Of course, in our case the origin of this contribution is related with the SU(3) flavour symmetry breaking.

Let us now express the mixing angles $\vartheta_{8}, \vartheta_{0}$ in terms of one mixing angle $\theta_{p}$ and quark masses. One has

$$
\begin{aligned}
& \tan \vartheta_{8}=\frac{f_{\eta^{\prime}}^{8}}{f_{\eta}^{8}}=\tan \left(\theta_{p}-\arctan \frac{\sqrt{2}\left(M_{s}-M_{u}\right)}{M_{u}+2 M_{s}}\right), \\
& \tan \vartheta_{0}=-\frac{f_{\eta}^{0}}{f_{\eta^{\prime}}^{0}}=\tan \left(\theta_{p}+\arctan \frac{\sqrt{2}\left(M_{s}-M_{u}\right)}{2 M_{u}+M_{s}}\right) .
\end{aligned}
$$

It follows then that:

$$
\begin{aligned}
& \vartheta_{8}=\psi-\arctan \left(\sqrt{2} \frac{M_{s}}{M_{u}}\right), \\
& \vartheta_{0}=\psi-\arctan \left(\sqrt{2} \frac{M_{u}}{M_{s}}\right),
\end{aligned}
$$

where $\psi=\theta_{p}+\arctan \sqrt{2}$. Similar formulae have been obtained in [39] by Feldmann, Kroll, and Stech. One should not confuse the angle $\theta_{p}$, defined by the rotation (53) and contributing to the matrix $f_{P}^{a}$ as it is shown in Eq. (54), with the naive identification $\vartheta_{8}=\vartheta_{0}=\theta_{p}$ discussed in the literature in connection with the one mixing angle problem. Our consideration is perfectly consistent with the two mixing angles approach.

\subsection{Strange-nonstrange basis for decay couplings}

The axial-vector currents can be taken in a different basis, namely, we shall consider now the nonstrange $\mathcal{A}_{\mu}^{\mathrm{ns}}$ and strange $\mathcal{A}_{\mu}^{s}$ currents

$$
\mathcal{A}_{\mu}^{\mathrm{ns}}=\sqrt{\frac{2}{3}} \mathcal{A}_{\mu}^{0}+\frac{1}{\sqrt{3}} \mathcal{A}_{\mu}^{8}, \quad \mathcal{A}_{\mu}^{s}=\frac{1}{\sqrt{3}} \mathcal{A}_{\mu}^{0}-\sqrt{\frac{2}{3}} \mathcal{A}_{\mu}^{8} .
$$

The singlet $\phi_{R}^{0}$ and octet $\phi_{R}^{8}$ fields are also rotated to the new basis

$$
\left(\begin{array}{c}
\phi^{\mathrm{ns}} \\
\phi^{s}
\end{array}\right)=\frac{1}{\sqrt{3}}\left(\begin{array}{cc}
\sqrt{2} & 1 \\
1 & -\sqrt{2}
\end{array}\right)\left(\begin{array}{c}
\phi_{R}^{0} \\
\phi_{R}^{8}
\end{array}\right)
$$

The corresponding matrix elements are easily calculated

$$
\left\langle 0\left|\mathcal{A}_{\mu}^{\mathrm{ns}}(0)\right| \phi^{\mathrm{ns}}(p)\right\rangle=\mathrm{i} p_{\mu} \frac{M_{u}}{g}, \quad\left\langle 0\left|\mathcal{A}_{\mu}^{s}(0)\right| \phi^{s}(p)\right\rangle=\mathrm{i} p_{\mu} \frac{M_{s}}{g} .
$$

The physical states $P=\eta, \eta^{\prime}$ are the mixtures of the nonstrange and strange components, this follows from Eqs. (53) and (66) 


$$
\left(\begin{array}{c}
\phi^{\mathrm{ns}} \\
\phi^{s}
\end{array}\right)=\left(\begin{array}{cc}
\cos \psi & \sin \psi \\
-\sin \psi & \cos \psi
\end{array}\right)\left(\begin{array}{c}
\eta \\
\eta^{\prime}
\end{array}\right)
$$

where the angle $\psi=\theta_{p}+\arctan \sqrt{2} \simeq \theta_{p}+54.74^{\circ}$.

Next one can find the couplings describing decays of physical states in the hadron vacuum

$$
\left\langle 0\left|\mathcal{A}_{\mu}^{i}(0)\right| P(p)\right\rangle=\mathrm{i} f_{P}^{i} p_{\mu}, \quad(i=\mathrm{ns}, s) .
$$

The result can be represented in a way which is similar to the one of Leutwyler-Kaiser [40]

$$
\left\{f_{P}^{i}\right\}=\left(\begin{array}{ll}
f_{\eta}^{\mathrm{ns}} & f_{\eta}^{s} \\
f_{\eta^{\prime}}^{\mathrm{ns}} & f_{\eta^{\prime}}^{s}
\end{array}\right)=\left(\begin{array}{cc}
f_{\mathrm{ns}} \cos \vartheta_{\mathrm{ns}} & -f_{s} \sin \vartheta_{s} \\
f_{\mathrm{ns}} \sin \vartheta_{\mathrm{ns}} & f_{s} \cos \vartheta_{s}
\end{array}\right) .
$$

Our calculations show that

$$
f_{\eta}^{\mathrm{ns}}=\frac{M_{u}}{g} \cos \psi, \quad f_{\eta}^{s}=-\frac{M_{s}}{g} \sin \psi, \quad f_{\eta^{\prime}}^{\mathrm{ns}}=\frac{M_{u}}{g} \sin \psi, \quad f_{\eta^{\prime}}^{s}=\frac{M_{s}}{g} \cos \psi .
$$

It follows that the basic parameters $f_{\mathrm{ns}}, f_{s}, \vartheta_{\mathrm{ns}}, \vartheta_{s}$ of the matrix $\left\{f_{P}^{i}\right\}$, being expressed in terms of model parameters (in the approximation considered), are

$$
f_{\mathrm{ns}}=\frac{M_{u}}{g}=f_{\pi}, \quad f_{s}=\frac{M_{s}}{g}, \quad \psi=\vartheta_{\mathrm{ns}}=\vartheta_{s} .
$$

There is a direct relation between a common mixing angle $\vartheta_{\mathrm{ns}}=\vartheta_{s}$ and the OZI-rule which has been discussed in [39-41].

\subsection{Mass formulae and the mixing angle $\theta_{p}$}

The gap equations are an essential ingredient to obtain mass formulae of pseudoscalars. One obtains them equating to zero the tadpole contributions from the Lagrangian $\mathcal{L}_{\text {bos }}$, see Eq. (45)

$$
\left\{\begin{array}{l}
h_{u}+\frac{N_{c}}{6 \pi^{2}} M_{u}\left(3 I_{0}-\Delta_{u s} I_{1}\right)=0 \\
h_{s}+\frac{N_{c}}{6 \pi^{2}} M_{s}\left(3 I_{0}+2 \Delta_{u s} I_{1}\right)=0
\end{array}\right.
$$

The stationary phase equations (143) taken in the isospin limit $\left(m_{u}=m_{d}\right)$ have been also used to obtain the mass spectrum ${ }^{6}$

$$
\begin{aligned}
m_{\pi}^{2} & =\left(\frac{g^{2} m_{u}}{G M_{u}}\right) \frac{1}{1+\omega_{s}+\rho+\tau_{u u}}, \\
m_{K}^{2} & =\frac{g^{2}}{G}\left(\frac{m_{u}+m_{s}}{M_{u}+M_{s}}\right) \frac{1}{1+\omega_{u}+\rho+\tau_{u u}+\tau_{s s}-\tau_{u s}} \\
m_{\eta_{\mp}}^{2} & =\frac{g^{2}}{2}\left(A+B \mp \sqrt{(A-B)^{2}+4 D^{2}}\right) .
\end{aligned}
$$

Here $\eta_{-}=\eta, \eta_{+}=\eta^{\prime}$

\footnotetext{
${ }^{6}$ See Eq. (148) for our notations of $\omega_{i}, \rho$, and $\tau_{i j}$.
} 
There is a mixing between the $(0,8)$ states in the multiplet. The symmetric mass matrix $\mathcal{M}_{p}$ is given by

$$
\mathcal{M}_{p}=\frac{g^{2}}{2}\left(\phi_{R}^{0}, \phi_{R}^{8}\right)\left(\begin{array}{ll}
A & D \\
D & B
\end{array}\right)\left(\begin{array}{c}
\phi_{R}^{0} \\
\phi_{R}^{8}
\end{array}\right),
$$

where we have

$$
\begin{aligned}
& A+B=\frac{h_{u}}{M_{u}}+\frac{h_{s}}{M_{s}}+\frac{2(1+\rho)-\omega_{s}+\tau_{u u}+\tau_{s s}}{G \operatorname{det} N^{(2)}}, \\
& A-B=\frac{1}{3}\left(\frac{h_{u}}{M_{u}}-\frac{h_{s}}{M_{s}}+\frac{8 \omega_{u}+\omega_{s}+\tau_{s s}-\tau_{u u}}{G \operatorname{det} N^{(2)}}\right), \\
& D=\frac{\sqrt{2}}{3}\left(\frac{h_{u}}{M_{u}}-\frac{h_{s}}{M_{s}}+\frac{\omega_{s}-\omega_{u}+\tau_{s s}-\tau_{u u}}{G \operatorname{det} N^{(2)}}\right) .
\end{aligned}
$$

The non-diagonal term of the matrix vanishes in the SU(3) flavour symmetric case $\left(m_{u}=m_{d}=m_{s}\right.$ ), otherwise $D \neq 0$. This matrix is diagonalized by an orthogonal transformation (53) to the states $\left(\eta, \eta^{\prime}\right)$ with masses given by Eq. (76). The singlet-octet mixing angle is

$$
\tan 2 \theta_{p}=\frac{2 D}{A-B}
$$

It is easily seen from Eq. (81) that the mixing angle $\theta_{p}$ is equal to its ideal value: $\tan \left(2 \theta_{\text {id }}\right)=2 \sqrt{2}$, i.e., $\theta_{\text {id }} \simeq 35.26^{\circ}$ and $\psi=90^{\circ}$, if $\kappa=0$. As a result we have $\eta \propto \phi^{\text {ns }}$ and $\eta^{\prime} \propto \phi^{s}$, thus the eight-quark interactions have no influence on the flavour content of $\eta, \eta^{\prime}$ without the 't Hooft term.

Consider now the $\eta-\eta^{\prime}$ masses (76) presented as follows:

$$
m_{\eta_{\mp}}^{2}=m_{K}^{2}+Q_{1} \mp \sqrt{\left(m_{K}^{2}-m_{\pi}^{2}-Q_{2}\right)^{2}+2 Q_{3}^{2}} .
$$

The independent functions $Q_{1}, Q_{2}$, and $Q_{3}$ are equal to

$$
\begin{aligned}
Q_{1}= & \frac{g^{2}\left(2 \omega_{u}+\omega_{s}+\tau_{u u}+\tau_{s s}-2 \tau_{u s}\right)}{2 G \operatorname{det} N^{(2)}}-\left(M_{s}-M_{u}\right)^{2} \\
& -\frac{2 g^{2}\left(\omega_{u}-\tau_{u s}\right)\left(\omega_{s}-\omega_{u}+\tau_{u u}+\tau_{s s}-2 \tau_{u s}\right)}{G\left(1+\rho+\omega_{u}+\tau_{u u}+\tau_{s s}-\tau_{u s}\right) \operatorname{det} N^{(2)}}, \\
Q_{2}= & \frac{g^{2}\left(\omega_{s}+\tau_{s s}-\tau_{u u}\right)}{2 G \operatorname{det} N^{(2)}}+\left(M_{s}-M_{u}\right)^{2} \\
& +\frac{g^{2}\left(\omega_{s}-\omega_{u}+\tau_{u s}-\tau_{s s}\right)}{G\left(1+\rho+\omega_{s}+\tau_{u u}\right)\left(1+\rho+\omega_{u}+\tau_{u u}+\tau_{s s}-\tau_{u s}\right)}, \\
Q_{3}= & \frac{g^{2} \omega_{u}}{G \operatorname{det} N^{(2)}} .
\end{aligned}
$$

\footnotetext{
${ }^{7}$ Note that the orthogonal transformation (53) is written for $\kappa \neq 0$, what corresponds to $A-B>0$. If $\kappa=0$, one has the opposite inequality $A-B<0$, and, as a consequence, one should replace in Eqs. (53) and (68) the fields $\eta \leftrightarrow \eta^{\prime}$.
} 
In the large- $N_{\mathrm{c}}$ approximation $\left(Q_{1}, Q_{2}, Q_{3} \rightarrow Q_{1}^{\mathrm{LO}}, Q_{2}^{\mathrm{LO}}, Q_{3}^{\mathrm{LO}}\right)$ these functions are related to each other and to the ghost coupling $\lambda_{\eta}$, introduced by Veneziano in [6],

$$
\frac{2}{3} Q_{1}^{\mathrm{LO}}=2 Q_{2}^{\mathrm{LO}}=Q_{3}^{\mathrm{LO}}=\frac{g^{2} \omega}{G}=\frac{\lambda_{\eta}^{2}}{N_{\mathrm{c}}} \quad\left(\text { large } N_{\mathrm{c}}, S U(3) \text { limit }\right)
$$

where $\omega \sim 1 / N_{\mathrm{c}}$ is a leading order contribution of $\omega_{u}$, or $\omega_{s}$. We have for $\lambda_{\eta}^{2}$

$$
\lambda_{\eta}^{2}=-\left.\frac{\kappa N_{\mathrm{c}}}{16 f_{\pi}^{2}}\left(\frac{M}{G}\right)^{3}\right|_{N_{\mathrm{c}} \rightarrow \infty} .
$$

With these specific values of $Q$ 's our expressions for the masses of $\eta, \eta^{\prime}$ mesons coincide with Eq. (34) of Veneziano work in [6]. Moreover, the Witten-Veneziano formula for the mass of $\eta^{\prime}$,

$$
m_{\eta^{\prime}}^{2}+m_{\eta}^{2}-2 m_{K}^{2}=\frac{3}{N_{\mathrm{c}}} \lambda_{\eta}^{2}=-\left.\frac{6}{f_{\pi}^{2}} \chi(0)\right|_{\mathrm{YM}},
$$

which is obtained in the large $N_{\mathrm{c}}$ limit of QCD for non-vanishing quark masses, relates the $\eta^{\prime}$ mass with the topological susceptibility in pure Yang-Mills theory $\left.\chi(0)\right|_{\mathrm{YM}}$. Consequently, Eq. (87) yields

$$
\left.\chi(0)\right|_{\mathrm{YM}}=\frac{\kappa}{4}\left(\frac{M}{2 G}\right)^{3} \quad\left(\text { large } N_{\mathrm{c}}\right) .
$$

The interesting feature here is the explicit demonstration that the eight-quark forces contribute to Eqs. (83)-(85) in such a way that the only dominant term in (88) is still the 't Hooft interaction, even though the $\rho_{i j}$, $\tau_{i j}$ may formally be of the same $1 / N_{c}$-order as $\omega_{i}$.

One can use the most recent lattice calculation of the topological susceptibility in [42]: $\left.\chi(0)\right|_{\mathrm{YM}}=-(1.33 \pm 0.14) \times 10^{-3} \mathrm{GeV}^{4}$ to find $Q_{2}^{\mathrm{LO}}=0.156 \mathrm{GeV}^{2}$. Additionally, we have at leading $N_{\mathrm{c}}$-order

$$
\tan 2 \theta_{p}=2 \sqrt{2} \frac{m_{K}^{2}-m_{\pi}^{2}-Q_{2}+\frac{1}{2} Q_{3}}{m_{K}^{2}-m_{\pi}^{2}-Q_{2}-4 Q_{3}} \rightarrow 2 \sqrt{2} \frac{m_{K}^{2}-m_{\pi}^{2}}{m_{K}^{2}-m_{\pi}^{2}-9 Q_{2}^{\mathrm{LO}}},
$$

obtaining approximately a mixing angle $\theta_{p} \simeq-14^{\circ}$, and masses $m_{\eta} \simeq 516 \mathrm{MeV}$, $m_{\eta^{\prime}} \simeq 1077 \mathrm{MeV}$. These numbers reflect the general picture presented in Section 5 rather well.

\section{Scalars: masses and the mixing angle $\theta_{s}$}

\subsection{Mass spectrum of the scalar nonet}

The masses of the scalar nonet: $a_{0}(I=1), K_{0}^{*}(I=1 / 2), f_{0}^{\mp}(I=0)$, are

$$
\begin{aligned}
& m_{a_{0}}^{2}=m_{\pi}^{2}+4 M_{u}^{2}+\frac{2 g^{2}\left(\omega_{s}-\tau_{u u}\right)}{G\left[\left(1+\rho+2 \tau_{u u}\right)^{2}-\left(\omega_{s}-\tau_{u u}\right)^{2}\right]}, \\
& m_{K_{0}^{*}}^{2}=m_{K}^{2}+4 M_{u} M_{s}+\frac{2 g^{2}\left(\omega_{u}-\tau_{u s}\right)}{G\left[\left(1+\rho+\tau_{u u}+\tau_{s s}\right)^{2}-\left(\omega_{u}-\tau_{u s}\right)^{2}\right]}, \\
& m_{f_{0}^{\mp}}^{2}=\frac{g^{2}}{2}\left(\mathcal{A}+\mathcal{B} \mp \sqrt{(\mathcal{A}-\mathcal{B})^{2}+4 \mathcal{D}^{2}}\right) .
\end{aligned}
$$


To obtain the last formula (93) the mass matrix of isospin singlets $\sigma_{R}^{0}$ and $\sigma_{R}^{8}$ in the Lagrangian (38)

$$
\mathcal{M}_{s}=\frac{g^{2}}{2}\left(\sigma_{R}^{0}, \sigma_{R}^{8}\right)\left(\begin{array}{ll}
\mathcal{A} & \mathcal{D} \\
\mathcal{D} & \mathcal{B}
\end{array}\right)\left(\begin{array}{l}
\sigma_{R}^{0} \\
\sigma_{R}^{8}
\end{array}\right),
$$

where

$$
\begin{aligned}
& \mathcal{A}+\mathcal{B}=\frac{h_{u}}{M_{u}}+\frac{h_{s}}{M_{s}}+\frac{N_{\mathrm{c}} I_{1}}{\pi^{2}}\left(M_{s}^{2}+M_{u}^{2}\right)+\frac{2+\omega_{s}+4 \rho+3\left(\tau_{u u}+\tau_{s s}\right)}{G \operatorname{det} N^{(1)}}, \\
& \mathcal{A}-\mathcal{B}=\frac{h_{u}}{M_{u}}-\frac{h_{s}}{M_{s}}-\frac{8 \omega_{u}+\omega_{s}+2\left(2 \rho_{u u}-\rho_{s s}+8 \rho_{u s}\right)+3\left(\tau_{u u}-\tau_{s s}\right)}{3 G \operatorname{det} N^{(1)}}, \\
& \mathcal{D}=\sqrt{2}\left(\frac{h_{u}}{M_{u}}-\frac{h_{s}}{M_{s}}-\frac{\omega_{s}-\omega_{u}+2\left(2 \rho_{u u}-\rho_{s s}-\rho_{u s}\right)+3\left(\tau_{u u}-\tau_{s s}\right)}{3 G \operatorname{det} N^{(1)}}\right),
\end{aligned}
$$

has been diagonalized by an orthogonal transformation

$$
\left(\begin{array}{c}
f_{0}^{-} \\
f_{0}^{+}
\end{array}\right)=\left(\begin{array}{cc}
\cos \theta_{s} & \sin \theta_{s} \\
-\sin \theta_{s} & \cos \theta_{s}
\end{array}\right)\left(\begin{array}{c}
\sigma_{R}^{0} \\
\sigma_{R}^{8}
\end{array}\right),
$$

with the angle given by

$$
\tan 2 \theta_{s}=\frac{2 \mathcal{D}}{\mathcal{A}-\mathcal{B}}
$$

Let us apply formula (96) to the case in which the 't Hooft determinant is neglected. We find that the mixing angle $\theta_{s} \neq \theta_{\text {id }}$ at $\kappa=0$. The reason is that the terms which are proportional to $\rho_{u s}$ in $\mathcal{D}$ and $\mathcal{A}-\mathcal{B}$ have different coefficients. Therefore the $f_{0}^{-}$meson has an admixture of the strange component and correspondingly the $f_{0}^{+}$meson has an admixture of nonstrange quarks due to the eight-quark interactions ${ }^{8}$ with coupling $g_{1}$. Such admixtures explicitly violate the OZI rule in these scalar channels.

We must notice that the singlet-octet splitting of scalars is more sensitive to the eightquark interactions, as opposed to the pseudoscalar case considered above. We can gain some understanding of this by writing slightly less explicit formulae for scalars. For that we turn again to the large $N_{\mathrm{c}}$ arguments, postponing the exact calculations till Section 5 .

\subsection{The $1 / N_{c}$ consideration}

The $1 / N_{\mathrm{c}}$ expansion is usually a good approximation for hadrons. If we accept this idea, we can deduce from the above formulae a clear qualitative picture of the role played by the eightquark forces in the mass spectra of scalars. Our starting point are the following expressions:

$$
\begin{aligned}
& m_{a_{0}}^{2}=m_{\pi}^{2}+4 M_{u}^{2}+\frac{2 g^{2}}{G}\left(\omega_{s}-\tau_{u u}\right)+\cdots \\
& m_{K_{0}^{*}}^{2}=m_{K}^{2}+4 M_{u} M_{s}+\frac{2 g^{2}}{G}\left(\omega_{u}-\tau_{u s}\right)+\cdots \\
& m_{f_{0}^{\mp}}^{2}=m_{K}^{2}+\left(M_{s}+M_{u}\right)^{2}+\mathcal{Q}_{1} \mp \sqrt{\left(\Delta_{K \pi}+\mathcal{Q}_{2}\right)^{2}+8\left(\Delta_{K \pi}+\mathcal{Q}_{3}\right)^{2}},
\end{aligned}
$$

\footnotetext{
${ }^{8}$ We have $f_{0}^{-} \propto(\bar{u} u+\bar{d} d)$ and $f_{0}^{+} \propto \bar{s} s$ at $\kappa=g_{1}=0$.
} 
where $\Delta_{K \pi}=m_{K}^{2}-m_{\pi}^{2}$, and ellipses are used to denote omitted terms of $1 / N_{\mathrm{c}}^{2}$ order and higher. The functions $\mathcal{Q}_{1}, \mathcal{Q}_{2}$, and $\mathcal{Q}_{3}$ are

$$
\begin{aligned}
& \mathcal{Q}_{1}=\frac{g^{2}}{2 G}\left[2 \omega_{u}-\omega_{s}-2 \rho-\left(\tau_{u u}+\tau_{s s}+2 \tau_{u s}\right)\right]+\cdots, \\
& \mathcal{Q}_{2}=-Q_{2}^{\mathrm{LO}}+\frac{g^{2}}{3 G}\left(4 \omega_{u}+2 \omega_{s}+2 \rho_{u u}-\rho_{s s}+8 \rho_{u s}\right)+\cdots, \\
& \mathcal{Q}_{3}=-Q_{2}^{\mathrm{LO}}+\frac{g^{2}}{3 G}\left(2 \omega_{s}-\frac{\omega_{u}}{2}+2 \rho_{u u}-\rho_{s s}-\rho_{u s}\right)+\cdots
\end{aligned}
$$

The contributions from the different $\tau$ 's are exactly canceled in Eqs. (101), (102) at this order. To simplify our numerical estimations, let us also neglect the $S U(3)$ breaking effects in the $\mathcal{Q}_{\mathrm{i}}$. Thus, one has to lowest order in $1 / N_{c}$ and in the $S U(3)$ limit

$$
\mathcal{Q}_{1}^{\mathrm{LO}}=Q_{2}^{\mathrm{LO}}-3 E_{1}^{\mathrm{LO}}-2 E_{2}^{\mathrm{LO}}, \quad \mathcal{Q}_{2}^{\mathrm{LO}}=3\left(Q_{2}^{\mathrm{LO}}+E_{1}^{\mathrm{LO}}\right), \quad \mathcal{Q}_{3}^{\mathrm{LO}}=0,
$$

where the eight-quark contributions $E_{1}^{\mathrm{LO}}$ and $E_{2}^{\mathrm{LO}}$, namely

$$
E_{1}^{\mathrm{LO}}=\left.\frac{g^{2} \rho_{u u}}{G}\right|_{\operatorname{large} N_{\mathrm{c}}}, \quad E_{2}^{\mathrm{LO}}=\left.\frac{g^{2} \tau_{u u}}{G}\right|_{\text {large } N_{\mathrm{c}}},
$$

are proportional to the strengths $\sim g_{1}$ and $g_{2}$ correspondingly.

Next, let us try to understand in simple terms the hierarchy inside the nonet. It is easy to see from Eqs. (97)-(99) that $m_{f_{0}^{-}}<m_{a_{0}}<m_{K_{0}^{*}}<m_{f_{0}^{+}}$. This is in an agreement with the result of analysis [43]. Let us do some crude numerical estimates. We have at leading order ${ }^{9}$

$$
\begin{aligned}
& m_{K_{0}^{*}}^{2}-m_{f_{0}^{\mp}}^{2}=\mathcal{Q}_{2}^{\mathrm{LO}} \pm \sqrt{\left(\Delta_{K \pi}+\mathcal{Q}_{2}^{\mathrm{LO}}\right)^{2}+8 \Delta_{K \pi}^{2}}, \\
& m_{K_{0}^{*}}^{2}-m_{a_{0}}^{2}=\Delta_{K \pi}+4 M_{u}\left(M_{s}-M_{u}\right), \\
& \Delta_{K \pi}=2 M_{s}\left(M_{s}-M_{u}\right) .
\end{aligned}
$$

First, we conclude that eight-quark forces are probably unimportant for the difference $m_{K_{0}^{*}}^{2}-m_{a_{0}}^{2}$.

Second, we use the ratio $f_{K} / f_{\pi}=1.22$ to find $M_{s} / M_{u}$. Indeed, it follows from Eqs. (51), (56), (57), (63) and (64) that

$$
\begin{aligned}
& \frac{f_{K}}{f_{\pi}}=\frac{1}{2}\left(1+\frac{M_{s}}{M_{u}}\right), \\
& \frac{f_{8}}{f_{\pi}}=\sqrt{\frac{1}{3}\left(1+2 \frac{M_{s}^{2}}{M_{u}^{2}}\right)}, \quad \frac{f_{0}}{f_{\pi}}=\sqrt{\frac{1}{3}\left(2+\frac{M_{s}^{2}}{M_{u}^{2}}\right)} \\
& \vartheta_{0}-\vartheta_{8}=\arctan \left[\frac{\sqrt{2}}{3}\left(\frac{M_{s}}{M_{u}}-\frac{M_{u}}{M_{s}}\right)\right] .
\end{aligned}
$$

${ }^{9}$ The last Eq. (107) follows from our general result Eqs. (74), (75) in the pseudoscalar sector for the difference

$$
m_{K}^{2}-m_{\pi}^{2}=2 M_{s}\left(M_{s}-M_{u}\right)+\frac{g^{2}\left(\omega_{s}-\omega_{u}+\tau_{u s}-\tau_{s s}\right)}{G\left(1+\omega_{s}+\rho+\tau_{u u}\right)\left(1+\omega_{u}+\rho+\tau_{u u}+\tau_{s s}-\tau_{u s}\right)} .
$$


One easily finds $M_{s} / M_{u}=1.44$. Numerically, this yields $f_{8}=1.31 f_{\pi}, f_{0}=1.17 f_{\pi}$, $\vartheta_{0}-\vartheta_{8}=19.5^{\circ}$.

Third, we use phenomenological values $m_{\pi} \simeq 138 \mathrm{MeV}$ (averaged over the isotopic triplet $\pi^{0}, \pi^{ \pm}$) and $m_{K} \simeq 495.7 \mathrm{MeV}$ (averaged value for isotopic duplet $K^{+}, K^{0}$ ) to obtain $\Delta_{K \pi}=0.227 \mathrm{GeV}^{2}$, and $m_{K_{0}^{*}}^{2}-m_{a_{0}}^{2} \simeq 2.39 \Delta_{K \pi}=0.542 \mathrm{GeV}^{2}$.

In order to make more progress, we need a further dynamical input. For that we identify the quark-antiquark $a_{0}$-state of the model with the known $I^{G}\left(J^{\mathrm{PC}}\right)=1^{-}\left(0^{++}\right)$resonance $a_{0}(980)$. This resonance is often considered as $K \bar{K}$ molecular-like bound state [44]. The four-quark nature of the $a_{0}(980)$ meson is also widely discussed in the literature (see, e.g., the recent paper [45] and references therein), where $a_{0}(980)$ is a compact $K \bar{K}$ state. The extended molecule case does not exclude that the core part of the wave function may be dominantly $q \bar{q}$ [46]. The four-quark picture is however based essentially on the MIT-bag model, thus representing an alternative approach to the problem.

Now, by using that $m_{a_{0}(980)} \simeq 980 \mathrm{MeV}$, one derives $m_{K_{0}^{*}} \simeq 1226 \mathrm{MeV}$. We suppose that this state may be identified with the wide $I\left(J^{P}\right)=\frac{1}{2}\left(0^{+}\right)$resonance $K_{0}^{*}(800)$ : $m_{K_{0}^{*}(800)}=797 \pm 19 \pm 43 \mathrm{MeV}, \Gamma=410 \pm 43 \pm 87 \mathrm{MeV}$, reported in [47]; see also [4852], where further support for this low lying state is given.

Before we calculate the masses of the two $f_{0}^{\mp}$ states from Eq. (105), consider the singletoctet mixing angle $\theta_{s}$ which can be written very compactly in the large $N_{\mathrm{c}}$ world, viz.,

$$
\tan 2 \theta_{s} \simeq 2 \sqrt{2} \frac{m_{K}^{2}-m_{\pi}^{2}}{m_{K}^{2}-m_{\pi}^{2}+\mathcal{Q}_{2}^{\mathrm{LO}}} .
$$

If the 't Hooft interaction term $Q_{2}^{\mathrm{LO}}$ dominates over $E_{1}^{\mathrm{LO}}$ in $\mathcal{Q}_{2}^{\mathrm{LO}}$ (this is possible when $g_{1} \sim 1 / N_{\mathrm{c}}^{5}$; in this case $E_{1}^{\mathrm{LO}} \sim 1 / N_{\mathrm{c}}^{2} \ll Q_{2}^{\mathrm{LO}} \sim 1 / N_{\mathrm{c}}$ ), one easily finds that $\theta_{s} \simeq 21^{\circ}$ (we have used here the estimate $Q_{2}^{\mathrm{LO}}=0.156 \mathrm{GeV}^{2}$ obtained before Eq. (90)).

One immediately derives from Eq. (105) the masses of the singlet-octet mixed states $f_{0}^{\mp}$ : $m_{f_{0}^{-}} \simeq 300 \mathrm{MeV}$ and $m_{f_{0}^{+}} \simeq 1407 \mathrm{MeV}$.

There is a strong cancellation in the formula for the $f_{0}^{-}$-mass

$$
\begin{aligned}
m_{f_{0}^{-}}^{2} & \simeq m_{K_{0}^{*}}^{2}-3 Q_{2}^{\mathrm{LO}}-\sqrt{\left(\Delta_{K \pi}+3 Q_{2}^{\mathrm{LO}}\right)^{2}+8 \Delta_{K \pi}^{2}} \\
& \simeq(1.502-0.468-0.946=0.088) \mathrm{GeV}^{2} .
\end{aligned}
$$

As a consequence the result is very sensitive to the parameters of the model. We shall see later that the best fit of the pseudoscalar channel leads to the value $m_{f_{0}^{-}} \simeq 550-750 \mathrm{MeV}$. Therefore, the lowest mass scalar meson, $f_{0}^{-}$, is identified with $f_{0}(600)$. The Particle Data Group assigns to this resonance the mass $m_{f_{0}(600)}=400-1200 \mathrm{MeV}$, and the width $\Gamma=600-1000 \mathrm{MeV}$.

The state $f_{0}^{+}$agrees with the state $f_{0}(1370): \quad m_{f_{0}(1370)}=1200-1500 \mathrm{MeV}$ $(\Gamma=200-500 \mathrm{MeV})$. The lower state $f_{0}(980)$ with the same quantum numbers is too far away from the value following from Eq. (105), thus our estimate shows that $f_{0}(980)$ may be not a member of the scalar quark-antiquark nonet considered. ${ }^{10}$

\footnotetext{
${ }^{10}$ It is notorious that the low-lying scalars are still the subject of many studies. Our conclusion agrees with some other results $[44,45,53]$, but we are aware that the point requires an additional analysis of scalar decays to be definitive. In different approaches, based on a coupled channel analysis, the pole position may also be affected by closed channels, see e.g., [49].
} 
It is known that the $U(1)_{A}$ breaking produces an opposite in sign mass-squared splitting between the octet and the singlet for the scalar and pseudoscalar mesons. This can be embodied in the approximate sum rule $[43,54]$

$$
m_{\eta^{\prime}}^{2}+m_{\eta}^{2}-2 m_{K}^{2}+m_{f_{0}^{+}}^{2}+m_{f_{0}^{-}}^{2}-2 m_{K_{0}^{*}}^{2} \simeq 0 .
$$

The r.h.s. results from the exact cancellation between the $1 / N_{c}$ order terms induced by the 't Hooft interaction (see, for example, Eq. (88)). One can see that eight-quark interactions may contribute to the sum rule already at $1 / N_{\mathrm{c}}$ order, if the coupling $g_{1}$ counts as $1 / N_{\mathrm{c}}^{4}$. Indeed, one obtains

$$
m_{\eta^{\prime}}^{2}+m_{\eta}^{2}-2 m_{K}^{2}+m_{f_{0}^{+}}^{2}+m_{f_{0}^{-}}^{2}-2 m_{K_{0}^{*}}^{2}=-6 E_{1}^{\mathrm{LO}}+\mathcal{O}\left(\frac{1}{N_{c}^{2}}\right) .
$$

This term has a negative sign, decreasing the sum $m_{f_{0}^{-}}^{2}+m_{f_{0}^{+}}^{2}$. Let us note that in this case $g_{1}$ lowers the value of $m_{f_{0}^{-}}$and due to the fine tuning effect in Eq. (112) the octet-singlet splitting grows with increasing $g_{1}$ in the scalar nonet. Thus the above sum rule is a good illustration of the possible impact of the eight-quark OZI violating forces on the scalar mesons.

\section{Numerical results}

We collect the results of the exact numerical calculations for the mass-spectra, mixing angles and quark condensates in three tables. Input is denoted by an asterisk. Table 1 contains the seven parameters of the model $m_{u}, m_{s}, \Lambda, G, \kappa, g_{1}$, and $g_{2}$.

Sets $(a, b, c)$ and $(d, e, f)$, are each a block for which we compare the effect of the new parameters $g_{1}, g_{2}$ as follows. In the first line of each grouping we set $g_{1}, g_{2}$ to zero, and fit four of the remaining parameters $\left(m_{u}, m_{s}, G, \kappa\right)$ by fixing $m_{\pi}, m_{K}, f_{\pi}, f_{K}$; in the first set $\Lambda$ is fixed through $f_{0}^{-}$, in the second through $\eta^{\prime}$. The reason to fix the empirically not well-known mass of $f_{0}^{-}$is that it is the most sensitive to changes of the parameter $g_{1}$ (we remind our discussion of Eq. (112)). By fixing it, one reverts the situation and is able to detect the effects of $g_{1}, g_{2}$ on the other observables. In the last set one sees that by fixing the mass of $\eta^{\prime}$, it is $f_{0}^{-}$that monopolizes the value of $g_{1}$.

For all sets, except of course the case $g_{1}=g_{2}=0$, the stability conditions (14) are fulfilled.

Table 1

Parameters of the model: $m_{u}, m_{s}(\mathrm{MeV}), G\left(\mathrm{GeV}^{-2}\right), \Lambda(\mathrm{MeV}), \kappa\left(\mathrm{GeV}^{-5}\right), g_{1}$, and $g_{2}\left(\mathrm{GeV}^{-8}\right)$. We also show the corresponding values of constituent quark masses $M_{u}$ and $M_{s}(\mathrm{MeV})$

\begin{tabular}{lllllrrrrr}
\hline & $m_{u}$ & $m_{s}$ & $M_{u}$ & $M_{s}$ & \multicolumn{1}{l}{$\Lambda$} & $\mathrm{G}$ & $-\kappa$ & \multicolumn{1}{c}{$g_{1}$} & \multicolumn{1}{c}{$g_{2}$} \\
\hline $\mathrm{a}$ & 5.2 & 161 & 302 & 486 & 934 & 7.18 & 1122 & $0^{*}$ & $0^{*}$ \\
$\mathrm{~b}$ & 5.5 & 175 & 325 & 523 & 896 & 8.78 & 774 & $1000^{*}$ & $0^{*}$ \\
$\mathrm{c}$ & 5.4 & 173 & 322 & 519 & 900 & 8.57 & 822 & $1000^{*}$ & $-132^{*}$ \\
$\mathrm{~d}$ & 6.1 & 189 & 372 & 646 & 839 & 12.16 & 1082 & $0^{*}$ & $0^{*}$ \\
$\mathrm{e}$ & 6.1 & 189 & 372 & 646 & 839 & 11.28 & 1083 & $1500^{*}$ & 327.24 \\
$\mathrm{f}$ & 6.1 & 189 & 372 & 646 & 839 & 8.92 & 1083 & $6000^{*}$ & 327.24 \\
\hline
\end{tabular}


Table 2

The masses, weak decay constants of light pseudoscalars (in $\mathrm{MeV}$ ), the singlet-octet mixing angle $\theta_{\mathrm{p}}$ (in degrees), and the quark condensates $\langle\bar{u} u\rangle,\langle\bar{s} s\rangle$ expressed as usual by positive combinations in $\mathrm{MeV}$

\begin{tabular}{|c|c|c|c|c|c|c|c|c|c|}
\hline & $m_{\pi}$ & $m_{K}$ & $m_{\eta}$ & $m_{\eta^{\prime}}$ & $f_{\pi}$ & $f_{K}$ & $\theta_{\mathrm{p}}$ & $-\langle\bar{u} u\rangle^{\frac{1}{3}}$ & $-\langle\bar{s} s\rangle^{\frac{1}{3}}$ \\
\hline $\mathrm{a}$ & $138^{*}$ & $494^{*}$ & 525 & 1761 & $92^{*}$ & $120^{*}$ & -1 & 246 & 210 \\
\hline b & $138^{*}$ & $494^{*}$ & 486 & 968 & $92^{*}$ & $120^{*}$ & -12 & 242 & 199 \\
\hline c & $138^{*}$ & $494^{*}$ & 493 & 1023 & $92^{*}$ & $120^{*}$ & -10 & 242 & 200 \\
\hline d & $138^{*}$ & $494^{*}$ & 476 & $958^{*}$ & $92^{*}$ & $116^{*}$ & -14.4 & 233 & 184 \\
\hline e & $138^{*}$ & $494^{*}$ & 476 & $958^{*}$ & $92^{*}$ & $116^{*}$ & -14.4 & 233 & 184 \\
\hline $\mathrm{f}$ & $138^{*}$ & $494^{*}$ & 476 & $958^{*}$ & $92^{*}$ & $116^{*}$ & -14.4 & 233 & 184 \\
\hline
\end{tabular}

Table 3

The masses of the scalar nonet (in $\mathrm{MeV}$ ), and the corresponding singlet-octet mixing angle $\theta_{\mathrm{s}}$ (in degrees)

\begin{tabular}{cccccc}
\hline & $m_{a_{0}(980)}$ & $m_{K_{0}^{*}(800)}$ & $m_{f_{0}(600)}$ & $m_{f_{0}(1370)}$ & $\theta_{\mathrm{s}}$ \\
\hline $\mathrm{a}$ & 1262 & 1347 & $600^{*}$ & 1436 & 16 \\
$\mathrm{~b}$ & 945 & 1150 & $600^{*}$ & 1309 & 15 \\
$\mathrm{c}$ & 980 & 1176 & $600^{*}$ & 1326 & 21 \\
$\mathrm{~d}$ & 993 & 1217 & 754 & 1391 & 25 \\
$\mathrm{e}$ & $980^{*}$ & 1204 & 691 & 1374 & 23 \\
$\mathrm{f}$ & $980^{*}$ & 1204 & 559 & 1362 & 23 \\
\hline
\end{tabular}

Tables 2 and 3 contain the results to be compared with the phenomenological data. The first set shows drastic effects of the parameter $g_{1}$ on mass spectra: going from (a) to (b) the $\eta^{\prime}$ mass is reduced by $40 \%$, getting close to its empirical value $m_{\eta^{\prime}}=957.78 \pm 0.14 \mathrm{MeV}$, while the $\eta$ mass gets smaller by $7 \%$. The overall effect is a reduction of the gap between these two states, which can be translated into the smaller value of the parameter $\kappa$ in (b), as compared to the one in (a).

The $\eta-\eta^{\prime}$ splitting can be illustrated by the formula (82) which takes the form

$$
m_{\eta}^{2}=m_{0}^{2}-\frac{8\left(m_{K}^{2}-m_{\pi}^{2}\right)^{2}+3 c_{q}}{9\left(m_{\eta^{\prime}}^{2}-m_{0}^{2}\right)},
$$

where $m_{0}^{2}=\frac{1}{3}\left(4 m_{K}^{2}-m_{\pi}^{2}\right)$ is the Gell-Mann-Okubo result for the $\eta$-mass. The remainder originates in the repulsion of $\eta$ and $\eta^{\prime}$ and represents an $\mathrm{SU}(3)$ breaking effect of second order. The coefficient $c_{q}$ depends on the $Q$ 's given by Eqs. (83)-(85), namely, $c_{q}=2\left(m_{K}^{2}-m_{\pi}^{2}\right)\left(Q_{1}-3 Q_{2}\right)+3\left(Q_{2}^{2}-Q_{1}^{2}+2 Q_{3}^{2}\right)$. Formula (115) for $c_{q} \neq 0$ extends the Veneziano result [6] (see Eq. (34) there) by including the SU(3) breaking corrections stemming from the 't Hooft and eight-quark interactions.

Numerically $m_{0}=565 \mathrm{MeV}$ is just a little bit larger, to be compared with the phenomenological value $m_{\eta}=547.30 \pm 0.12 \mathrm{MeV}$. The Witten-Veneziano correction (the second term $\left.\sim\left(m_{K}^{2}-m_{\pi}^{2}\right)^{2}\right)$ is related to the topological susceptibility ${ }^{11}$ and is about four times larger than it is required. Considering set (b), we obtain $m_{\eta} \simeq 496 \mathrm{MeV}$. This value is now corrected by the six and eight-quark contributions collected in $c_{q}$. Unfortunately they work in the same direction and do not improve

$\overline{11}$ One can find details, in particular, in the first reference of [38]. 
the low result for $\eta$. Finally one finds the value $m_{\eta}=486 \mathrm{MeV}$. This is the general tendency in all sets.

Other effects are seen in the reduction of all scalar masses, except the input one, by $25 \%$ for $a_{0}, 14 \%$ for $K_{0}^{*}, 9 \%$ for $f_{0}^{+}$. In set (c) the effect of $g_{2}$, keeping the same $g_{1}$ as in set (b), is seen to increase all masses again. It has in this case a negative value, which is also allowed by the stability conditions. The condensates and scalar mixing angle remain comparable in $(a, b, c)$, the pseudoscalar angle $\theta_{p}$ increases in absolute value from set (a) to (b). Its value quoted in Table 2 is around $\theta_{p}=-12^{\circ}$ being in agreement with estimates of Veneziano [6] and more recent calculations in [55].

This angle is directly related by Eqs. (63) and (64) with two mixing angles seen in the singlet and the octet components of the decay constants. We find, for example, for set (c): $\vartheta_{8}=-21.6^{\circ}, \vartheta_{0}=3.5^{\circ}$.

There is some ambiguity about the definition of quark condensates if the chiral symmetry is explicitly broken by bare quark masses $m_{i}$. The values given in Table 2 are obtained by the subtraction of the expectation value of $\bar{q}_{i} q_{i}$ in the perturbative vacuum from its expectation in the true vacuum:

$$
\left\langle\bar{q}_{i} q_{i}\right\rangle=\frac{1}{2}\left(\left.h_{i}\right|_{\Delta_{i} \neq 0}-\left.h_{i}\right|_{\Delta_{i}=0}\right),
$$

which is the definition used in [10]. Let us recall the recent update of the light-quark condensate at a scale of $1 \mathrm{GeV}:\langle\bar{q} q\rangle(1 \mathrm{GeV})=-(242 \pm 15 \mathrm{MeV})^{3}$, where $\bar{q} q=(\bar{u} u+\bar{d} d) / 2$ represents the isospin average of the non-strage quarks [56]. The flavour breaking ratio is known to be $\langle\bar{s} s\rangle /\langle\bar{q} q\rangle=0.8 \pm 0.3[56]$.

The second set (d,e,f): we fix the $\eta^{\prime}$ mass to its empirical value, and chose also $f_{K}$ closer to experiment, keeping $m_{\pi}, m_{K}, f_{\pi}$ as in all other cases. In (e) we obtain $g_{2}$ through the mass of $a_{0}(980)$ and take $g_{1}$ arbitrarily. In the pseudoscalar sector this lowers slightly the value of the $\eta$-mass and changes a bit the mixing angle $\theta_{p}$.

The main effect is visible in the $f_{0}(600)$ mass: a further increase in $g_{1}$, set (f), decreases further its mass, leaving the remaining observables almost unaffected. The repulsion between the two isosinglet levels caused by the eight-quark interaction thus lowers the value of $m_{f_{0}(600)}$ by about $200 \mathrm{MeV}$.

There is no marked effect on the $K_{0}^{*}(800)$ state, which continues to lie above the $a_{0}(980)$ mass. The large $N_{c}$ result (106) protects the inequality $m_{K_{0}^{*}}>m_{a_{0}}$ for the members of the quark-antiquark octet, which finally holds in the general case.

To summarize, the effect of eight-quark interactions on the mass spectrum, vacuum decay couplings, and mixing angles is relatively small as long as general properties of the QCD vacuum (the values of the quark condensates and the topological susceptibility) are correctly reproduced.

\section{Conclusions}

The role played by eight-quark interactions in the long wavelength limit of QCD has been addressed in a systematic way. A full understanding of its impact on the vacuum and properties of the low-lying spin zero mesonic spectra has been achieved. As an important by-product also the results associated with the well-known four and six quark Lagrangians due to Nambu-Jona-Lasinio and 't Hooft, on the body of which the eightquark terms are attached, are classified and presented according to stability criteria of 
the effective potential (reviewed and illustrated in Section 2.2), the large $N_{\mathrm{c}}$ counting scheme, approximate sum rules for meson masses, $U_{A}(1)$ and flavor $\mathrm{SU}(3)$ breaking terms, and OZI-rule violation. This "dissection" allows not only for a complete understanding of the full result, (i.e., in the leading order stationary phase approximation to the functional integral), for which we also give analytical expressions, but also to compare with other relevant works in the field. These discussions and respective formulae accompany every main derived step within the full result and serve as a "hitchhiker's guide" to the mesons in the multi-quark "galaxy". 12

An issue of much interest is the two-angle analysis of the $\eta-\eta^{\prime}$ mixing and its relation with the standard one-angle diagonalization. We clarify some existing confusion in the literature by deriving in full detail in Sections 3.2 and 3.3 the connection and equivalence of the two methods within the Lagrangian considered.

The masses and splitting of the complete result for $\eta-\eta^{\prime}$ system are conveniently cast in the form of Eq. (115), which separates in a transparent way the leading Gell-Mann-Okubo contribution, the Witten-Veneziano correction and the second-order SU(3) flavor breaking corrections due to six and eight-quark terms. These latter ones have a positive sign for the parameter sets which yield good fits for the remaining pseudoscalar observables and therefore add a small correction to the already large Witten-Veneziano term, thus yielding a larger splitting than the empirically observed.

Concerning the scalar sector we show that there exists a mass hierarchy within the model considerations which is not conform with the present understanding of the empirical results.

These drawbacks, being the result of an exhaustive and consistent study, clearly indicate that effects not considered, such as meson loops, higher orders in the heat kernel expansion, and confining forces, might be at work.

We view therefore the main role of eight-quark forces considered as follows: (i) they are of vital importance for the stability of the ground state built from four and sixquark interactions. They restrict the choice of the couplings $G, \kappa, g_{1}, g_{2}$ to the rather narrow window of combinations given by Eq. (14). This is important, since combinations outside the allowed range can at instances even yield a better spectrum for the pseudoscalar mesons alone. It would be an erroneous result, attributing minor importance to the corrections of the kind not considered. (ii) They help in understanding the effects caused by the OZI-violating terms with coupling strength $g_{1}$, which affect quite strongly the splitting of the $f_{0}^{-}, f_{0}^{+}$scalars, mainly pushing down the lower state, due to the strong cancellations reported in Eq. (112). (iii) They may be also of importance in decays and scattering, not considered so far. (iv) They give a clear indication that an hierarchy of multi-quark interactions, with dominance of lower ones, is present. This corroborates with recent lattice calculations [57] of gluon correlators, where the lower ones also dominate.

\section{Acknowledgments}

This work has been supported by grants provided by Fundação para a Ciência e a Tecnologia, POCTI/FNU/50336/2003 and POCI/FP/63412/2005. This research is part

\footnotetext{
${ }^{12}$ Borrowed from "The ultimate hitchhiker's guide to the galaxy", by D. Adams.
} 
of the EU integrated infrastructure initiative Hadron Physics project under contract No.RII3-CT-2004-506078. A.A. Osipov also gratefully acknowledges the Fundaçã o Calouste Gulbenkian for financial support.

\section{Appendix A. Response equation (21)}

An algebraic system of nonlinear equations (21) has, in general, more than one admissible solution. Our specific problem requires only the knowledge of all isolated real roots. In order to find them suppose that the system has at least one real solution, i.e., the set $\left\{h_{a}\right\}$. Let us now manipulate with the equations at hand in such a way that we can guess some values from this set. If our guess is correct, the system reduces to a smaller one, which finally can be solved. It may happen however that the smaller system is incomplete and therefore has a continuum of solutions. Such cases have to be excluded, because they are possible if and only if some of the components $\Delta_{a}$ are constrained (see below). It should be recalled that $\Delta_{a}$ is a set of independent variables (for different values of $a$ ) which will be fixed only later with the help of the gap equations. Thus any restriction on $\Delta_{a}$ at this stage leads to an internal contradiction and the corresponding solutions must be rejected. There is another reason to exclude a continuum of solutions, namely the stationary phase method cannot be applied for such a case.

After these general remarks, let's consider important details. Eq. (21) yields the following set:

$$
\begin{aligned}
& \mu h_{1}+v\left(h_{4} h_{6}+h_{5} h_{7}\right)=0, \\
& \mu h_{2}+v\left(h_{5} h_{6}-h_{4} h_{7}\right)=0, \\
& \Delta_{3}+\mu h_{3}+\frac{v}{2}\left(h_{4}^{2}+h_{5}^{2}-h_{6}^{2}-h_{7}^{2}\right)=0, \\
& \rho h_{4}+v\left(h_{1} h_{6}-h_{2} h_{7}\right)=0, \\
& \rho h_{5}+v\left(h_{1} h_{7}+h_{2} h_{6}\right)=0 \\
& \sigma h_{6}+v\left(h_{1} h_{4}+h_{2} h_{5}\right)=0, \\
& \sigma h_{7}+v\left(h_{1} h_{5}-h_{2} h_{4}\right)=0, \\
& \Delta_{8}+\frac{1}{2}(\rho+\sigma) h_{8}+\frac{v}{\sqrt{3}}\left[h_{1}^{2}+h_{2}^{2}+h_{3}^{2}-\frac{1}{2}\left(h_{4}^{2}+h_{5}^{5}+h_{6}^{2}+h_{7}^{2}\right)\right]=0, \\
& \Delta_{0}+\xi h_{0}+\frac{\kappa}{32} \sqrt{\frac{2}{3}}\left(3 h_{0}^{2}-h_{a}^{2}\right)+\frac{g_{2}}{\sqrt{2}} h_{8}\left(h_{1}^{2}+h_{2}^{2}+h_{3}^{2}-\frac{h_{8}^{2}}{3}\right) \\
& \quad+\frac{g_{2}}{2 \sqrt{2}}\left[\left(h_{4}^{2}+h_{5}^{2}\right)\left(\sqrt{3} h_{3}-h_{8}\right)-\left(h_{6}^{2}+h_{7}^{2}\right)\left(\sqrt{3} h_{3}+h_{8}\right)\right] \\
& \quad+g_{2} \sqrt{\frac{3}{2}}\left[h_{1}\left(h_{4} h_{6}+h_{5} h_{7}\right)+h_{2}\left(h_{5} h_{6}-h_{4} h_{7}\right)\right]=0,
\end{aligned}
$$

where $\mu, v, \rho, \sigma$, and $\xi$ are defined as follows:

$$
\mu=G+\frac{\kappa}{8 \sqrt{6}}\left(\sqrt{2} h_{8}-h_{0}\right)+\frac{1}{2}\left(g_{1}+g_{2}\right) h_{a}^{2}+\frac{g_{2}}{2} h_{0}\left(h_{0}+2 \sqrt{2} h_{8}\right),
$$




$$
\begin{aligned}
& v=\frac{\kappa}{16}+g_{2} \sqrt{\frac{3}{2}} h_{0}, \quad \xi=G+\frac{1}{2}\left(g_{1}+2 g_{2}\right) h_{a}^{2}-\frac{2 g_{2}}{3} h_{0}^{2}, \\
& \rho=G-\frac{\kappa}{16}\left(\sqrt{\frac{2}{3}} h_{0}-h_{3}+\frac{h_{8}}{\sqrt{3}}\right)+\frac{1}{2}\left(g_{1}+g_{2}\right) h_{a}^{2}+\frac{g_{2}}{2} h_{0}\left(h_{0}+\sqrt{6} h_{3}-\sqrt{2} h_{8}\right), \\
& \sigma=G-\frac{\kappa}{16}\left(\sqrt{\frac{2}{3}} h_{0}+h_{3}+\frac{h_{8}}{\sqrt{3}}\right)+\frac{1}{2}\left(g_{1}+g_{2}\right) h_{a}^{2}+\frac{g_{2}}{2} h_{0}\left(h_{0}-\sqrt{6} h_{3}-\sqrt{2} h_{8}\right) .
\end{aligned}
$$

Due to these definitions one has

$$
\mu-\sigma=v\left(\sqrt{3} h_{8}+h_{3}\right), \quad \mu-\rho=v\left(\sqrt{3} h_{8}-h_{3}\right) .
$$

We start from the observation: if $\mu \neq 0$, one can multiply Eqs. (120)-(123) by $\mu$ and, using Eqs. (117)-(118), obtain

$$
\begin{aligned}
& h_{4}\left[\mu \rho-v^{2}\left(h_{6}^{2}+h_{7}^{2}\right)\right]=0, \\
& h_{5}\left[\mu \rho-v^{2}\left(h_{6}^{2}+h_{7}^{2}\right)\right]=0, \\
& h_{6}\left[\mu \sigma-v^{2}\left(h_{4}^{2}+h_{5}^{2}\right)\right]=0, \\
& h_{7}\left[\mu \sigma-v^{2}\left(h_{4}^{2}+h_{5}^{2}\right)\right]=0 .
\end{aligned}
$$

This leads to four possible alternatives:

1. $\mu \neq 0, \quad h_{4}=h_{5}=h_{6}=h_{7}=0$.

2. $\mu \neq 0, \quad h_{4}=h_{5}=0, \quad \sigma=0$.

3. $\mu \neq 0, \quad h_{6}=h_{7}=0, \quad \rho=0$.

4. $\mu \neq 0, \quad \mu \rho=v^{2}\left(h_{6}^{2}+h_{7}^{2}\right), \quad \mu \sigma=v^{2}\left(h_{4}^{2}+h_{5}^{2}\right)$.

If $\mu=0$, then Eqs. (117)-(118) now imply other four alternatives:

5. $\mu=0, \quad v=0$.

6. $\mu=0, \quad v \neq 0, \quad h_{4}=h_{5}=0$.

7. $\mu=0, \quad v \neq 0, \quad h_{6}=h_{7}=0$.

8. $\mu=0, \quad v \neq 0, \quad h_{4}=h_{5}=h_{6}=h_{7}=0$.

Cases 2 and 3, as well as cases 6 and 7, are correlated. The existence of such correlation becomes clear if one notes the invariants of Eqs. (117)-(125) under the substitutions $h_{4} \leftrightarrow h_{7}, h_{5} \leftrightarrow h_{6}, h_{3} \leftrightarrow-h_{3}, \Delta_{3} \leftrightarrow-\Delta_{3}$. In particular these substitutions change $\rho \leftrightarrow \sigma$, leaving $\mu$ and $v$ without changes.

Case 1. In this case Eqs. (117) and (118) yield $h_{1}=h_{2}=0$ and one obtains a set of three equations to determine $h_{0}, h_{3}, h_{8}$. To make further progress one should switch to the flavour basis $0,3,8 \rightarrow u, d, s$, where one has 


$$
\left\{\begin{array}{l}
G h_{u}+\Delta_{u}+\frac{\kappa}{16} h_{d} h_{s}+\frac{g_{1}}{4} h_{u}\left(h_{u}^{2}+h_{d}^{2}+h_{s}^{2}\right)+\frac{g_{2}}{2} h_{u}^{3}=0 \\
G h_{d}+\Delta_{d}+\frac{\kappa}{16} h_{u} h_{s}+\frac{g_{1}}{4} h_{d}\left(h_{u}^{2}+h_{d}^{2}+h_{s}^{2}\right)+\frac{g_{2}}{2} h_{d}^{3}=0 \\
G h_{s}+\Delta_{s}+\frac{\kappa}{16} h_{u} h_{d}+\frac{g_{1}}{4} h_{s}\left(h_{u}^{2}+h_{d}^{2}+h_{s}^{2}\right)+\frac{g_{2}}{2} h_{s}^{3}=0 .
\end{array}\right.
$$

This system has been studied in our work [18].

Case 2. Again Eqs. (117)-(118) yield $h_{1}=h_{2}=0$. Then Eqs. (119) and (124) give $\Delta_{3}=\sqrt{3} \Delta_{8}$ (with the use of Eqs. (130)and $\sigma=0$ ). Since the variables $\Delta_{a}$ are supposed to be independent, one concludes that this result leads to an apparent contradiction. It may be also noted that the pair of quantities $\left(\Delta_{3}, \Delta_{8}\right)$ define in general a plane in the nonet space, if neither quantity vanishes. The found correlation means that only one axis is defined, in which case the system is not complete and the solution is underdetermined.

Case 3. As before Eqs. Eqs. ((117)-(118) yield $h_{1}=h_{2}=0$. Then Eqs. (119) and (124) with the use of Eqs. (130) and $\rho=0$ give $\Delta_{3}=-\sqrt{3} \Delta_{8}$. One comes anew to the abovementioned contradiction.

Case 4. From Eqs. (4) one obtains $\mu(\rho-\sigma)=v^{2}\left(h_{6}^{2}+h_{7}^{2}-h_{4}^{2}-h_{5}^{2}\right)$. Next, note that one can use Eqs. (130) to rewrite the result as follows:

$$
v\left[2 \mu h_{3}+v\left(h_{4}^{2}+h_{5}^{2}-h_{6}^{2}-h_{7}^{2}\right)\right]=0 .
$$

Since $v \neq 0$ this yields $\Delta_{3}=0$. (The equality $v=0$ would lead here to $\rho=\sigma=0$, and finally due to Eqs. (130) to $\mu=0$. The obtained contradiction proves that $v \neq 0$ ). Eqs. (117)-(118), as well as equations (4), imply that $\rho \sigma=v^{2}\left(h_{1}^{2}+h_{2}^{2}\right)$. Using this result and Eqs. (130), one can show that $\Delta_{8}=0$. It is clear that in this case the solutions suffer from the same kind of defects just mentioned above.

Case 5. Since $\mu=v=0$, Eq. (130) gives $\rho=\sigma=0$. This yields $\Delta_{3}=0$ and $\Delta_{8}=0$. This system is incomplete.

Case 6. Eqs. (122)-(123) yield $\sigma=0$. Since $\mu=\sigma=0$, Eq. (130) gives $h_{3}+\sqrt{3} h_{8}=0$. If $h_{6}$ and $h_{7}$ are nonzero, then from Eqs. (120) and (121), $h_{1}^{2}+h_{2}^{2}=0 \rightarrow h_{1}=h_{2}=0$, since we only allow real solutions. As a result, one obtains

$$
\Delta_{3}-\sqrt{3} \Delta_{8}=v h_{3}\left(h_{3}+\sqrt{3} h_{8}\right)=0 .
$$

Case 7. Eqs. (120) and (121) yield $\rho=0$. Next, due to $\sigma=0$, Eqs. (122) and (123) reduce to $h_{1} h_{4}+h_{2} h_{5}=0, h_{1} h_{5}-h_{2} h_{4}=0$, it follows then $h_{1}=h_{2}=0$. Eqs. (130) give $h_{3}=\sqrt{3} h_{8}$, that finally leads to the correlation $\Delta_{3}=-\sqrt{3} \Delta_{8}$.

Case 8. Eq. (119) reduces to $\Delta_{3}=0$. Eq. (124) has the form

$$
\Delta_{8}+\frac{v}{\sqrt{3}}\left(h_{1}^{2}+h_{2}^{2}+h_{3}^{2}-3 h_{8}^{2}\right)=0 .
$$

This case gives a new class of solutions. Nevertheless it can also be thrown out, as long as $\Delta_{3}=0$, by the same arguments as just given. 
One concludes that only the case 1 leads to the isolated real solutions of Eqs. (21) which must be taken into account. As has been shown in [18] one can choose the parameters of the model in such a way that only one real solution appears.

We would like to note that our investigation here recalls in many aspects the old results of Pais [34], who studied the response equations with octet driving forces. He dealt with the octet space of SU(3). Eqs. (21) have some more complicated structure, but the general conclusions remain true. Even the formal covariance property of the system under the transformation

$$
\begin{array}{ll}
h_{0}=q_{0}, & h_{1}=-q_{6}, \quad h_{2}=q_{7}, \quad h_{3}=\frac{1}{2}\left(q_{3}-\sqrt{3} q_{8}\right), \quad h_{4}=q_{4}, \quad h_{5}=q_{5}, \\
h_{6}=q_{1}, & h_{7}=-q_{2}, \quad h_{8}=-\frac{1}{2}\left(q_{8}+\sqrt{3} q_{3}\right)
\end{array}
$$

in the nonet space is fulfilled. This transformation brings Eq. (21) in the form

$$
b_{a}+G q_{a}+\frac{3 \kappa}{32} A_{a b c} q_{b} q_{c}+\frac{g_{1}}{2} q_{a} q_{b}^{2}+\frac{g_{2}}{2} d_{a b e} d_{c d e} q_{b} q_{c} q_{d}=0,
$$

where

$$
b_{3}=\frac{1}{2}\left(\Delta_{3}-\sqrt{3} \Delta_{8}\right), \quad b_{8}=-\frac{1}{2}\left(\Delta_{8}+\sqrt{3} \Delta_{3}\right),
$$

and all other $b_{a}=0$.

\section{Appendix B. Solving Eqs. (22) and (23) in the isotopic limit}

As it has been shown in Appendix A, the system (21) is actually reduced to three coupled equations (143) for three independent variables $h_{u}, h_{d}, h_{s}$. These variables are defined as follows $h_{a} \lambda_{a}=\operatorname{diag}\left(h_{u}, h_{d}, h_{s}\right)$, and easily related with $h_{0}, h_{3}$, and $h_{8}$, namely

$$
\begin{aligned}
& h_{u}=\frac{1}{\sqrt{3}}\left(\sqrt{2} h_{0}+\sqrt{3} h_{3}+h_{8}\right), \\
& h_{d}=\frac{1}{\sqrt{3}}\left(\sqrt{2} h_{0}-\sqrt{3} h_{3}+h_{8}\right), \\
& h_{s}=\sqrt{\frac{2}{3}}\left(h_{0}-\sqrt{2} h_{8}\right) .
\end{aligned}
$$

If we choose the current quark masses suitably, $m_{u}=m_{d} \neq m_{s}$, the flavour symmetry of the action is broken down to the isospin group $\mathrm{SU}(2)$. In this partial case one finds from Eq. (21) that $h_{a}=0$ for $a=1,2,3,4,5,6,7$, i.e., only two components $h_{0}$ and $h_{8}$ are generally nonzero. Thus, in virtue of Eq. (147) one concludes that $h_{u}=h_{d} \neq h_{s}$. We shall suppose that $h_{u}$ and $h_{s}$ are known. ${ }^{13}$

Let us solve Eqs. (22)-(23) to find the couplings $h_{a b}^{(1)}$ and $h_{a b}^{(2)}$ for the case with isospin symmetry. To represent the result we shall use dimensionless quantities

$$
\omega_{i}=\frac{\kappa h_{i}}{16 G}, \quad \rho_{i j}=\frac{g_{1} h_{i} h_{j}}{4 G}, \quad \tau_{i j}=\frac{g_{2} h_{i} h_{j}}{2 G}
$$

\footnotetext{
${ }^{13}$ See [18] for details, where a more general case, $h_{u} \neq h_{d} \neq h_{s}$, has been considered. The case studied here is a straightforward consequence of that result.
} 
induced by six- and eight-quark interactions with strength couplings $\kappa, g_{1}$, and $g_{2}$ correspondingly. We use also that $\rho=2 \rho_{u u}+\rho_{s s}$.

The result for $h_{a b}^{(1)}$ is

$$
h_{a b}^{(1)}= \begin{cases}\frac{-\delta_{a b}}{G\left(1-\omega_{s}+\rho+3 \tau_{u u}\right)} & (a, b=1,2,3), \\ \frac{-\delta_{a b}}{G\left(1-\omega_{u}+\rho+\tau_{u u}+\tau_{s s}+\tau_{u s}\right)} & (a, b=4,5,6,7), \\ \frac{-N_{a b}^{(1)}}{G \operatorname{det} N^{(1)}} & (a, b=0,8) .\end{cases}
$$

where the $2 \times 2$ symmetric matrix $N^{(1)}$ has elements

$$
\begin{aligned}
& N_{00}^{(1)}=1+\frac{1}{3}\left(\omega_{s}-4 \omega_{u}+10 \rho_{u u}+7 \rho_{s s}-8 \rho_{u s}\right)+\tau_{u u}+2 \tau_{s s}, \\
& N_{08}^{(1)}=N_{80}^{(1)}=\frac{\sqrt{2}}{3}\left[\omega_{u}-\omega_{s}+2\left(\rho_{s s}-2 \rho_{u u}+\rho_{u s}\right)+3\left(\tau_{s s}-\tau_{u u}\right)\right], \\
& N_{88}^{(1)}=1+\frac{1}{3}\left(4 \omega_{u}+2 \omega_{s}+14 \rho_{u u}+5 \rho_{s s}+8 \rho_{u s}\right)+2 \tau_{u u}+\tau_{s s},
\end{aligned}
$$

and its determinant is equal to

$$
\begin{aligned}
\operatorname{det} N^{(1)}= & 1+\omega_{s}-2 \omega_{u}^{2}+4 \rho \\
& +3\left[(1+\rho)\left(\tau_{u u}+\tau_{s s}\right)+\rho^{2}+\omega_{s}\left(\rho_{s s}-2 \rho_{u u}+\tau_{s s}\right)+3 \tau_{u s}^{2}+6 \tau_{u s} \rho_{u s}\right] .
\end{aligned}
$$

For $h_{a b}^{(2)}$ one obtains

$$
h_{a b}^{(2)}= \begin{cases}\frac{-\delta_{a b}}{G\left(1+\omega_{s}+\rho+\tau_{u u}\right)} & (a, b=1,2,3), \\ \frac{-\delta_{a b}}{G\left(1+\omega_{u}+\rho+\tau_{u u}+\tau_{s s}-\tau_{u s}\right)} & (a, b=4,5,6,7), \\ \frac{-N_{a b}^{(2)}}{G \operatorname{det} N^{(2)}} & (a, b=0,8) .\end{cases}
$$

where the $2 \times 2$ symmetric matrix $N^{(2)}$ has elements

$$
\begin{aligned}
& N_{00}^{(2)}=1+\frac{1}{3}\left(4 \omega_{u}-\omega_{s}\right)+\rho+\frac{1}{3}\left(\tau_{u u}+2 \tau_{s s}\right), \\
& N_{08}^{(2)}=N_{80}^{(1)}=\frac{\sqrt{2}}{3}\left(\omega_{s}-\omega_{u}+\tau_{s s}-\tau_{u u}\right), \\
& N_{88}^{(2)}=1-\frac{2}{3}\left(2 \omega_{u}+\omega_{s}\right)+\rho+\frac{1}{3}\left(2 \tau_{u u}+\tau_{s s}\right),
\end{aligned}
$$

and

$$
\operatorname{det} N^{(2)}=(1+\rho)\left(1+\rho+\tau_{u u}+\tau_{s s}-\omega_{s}\right)-2 \omega_{u}^{2}+\tau_{s s}\left(\tau_{u u}-\omega_{s}\right) .
$$




\section{References}

[1] S. Weinberg, Phys. A 96 (1979) 327;

J. Gasser, H. Leutwyler, Ann. Phys. (NY) 158 (1984) 142;

J. Gasser, H. Leutwyler, Nucl. Phys. B 250 (1985) 465.

[2] Y. Nambu, G. Jona-Lasinio, Phys. Rev. 122 (1961) 345, 124 (1961) 246; V.G. Vaks, A.I. Larkin, Zh. Éksp. Teor. Fiz. 40 (1961) 282, [Sov. Phys. JETP 13 (1961) 192]; B.A. Arbuzov, A.N. Tavkhelidze, R.N. Faustov, Dokl. Akad. Nauk SSSR 139 (1961) 345, [Sov. Phys. Dokl. 6 (1962) 598].

[3] E.V. Shuryak, Phys. Rep. 391 (2004) 381.

[4] Yu.A. Simonov, Phys. Lett. B412 (1977) 371; Yu.A. Simonov, Phys. Rev. D 65 (2002) 094018.

[5] G. 't Hooft, Phys. Rev. D 14 (1976) 3432; G. 't Hooft, Phys. Rev. D 18 (1978) 2199.

[6] E. Witten, Nucl. Phys. B 156 (1979) 269; G. Veneziano, Nucl. Phys. B 159 (1979) 213.

[7] P. Di Vecchia, Phys. Lett. B 85 (1979) 357;

P. Di Vecchia, G. Veneziano, Nucl. Phys. B 171 (1980) 253;

K. Kawarabayashi, N. Ohta, Nucl. Phys. B 175 (1980) 477.

[8] C. Rosenzweig, J. Schechter, C.G. Trahern, Phys. Rev. D 21 (1980) 3388.

[9] R. Alkofer, I. Zahed, Mod. Phys. Lett. A 4 (1989) 1737;

R. Alkofer, I. Zahed, Phys. Lett. B 238 (1990) 149.

[10] V. Bernard, R.L. Jaffe, U.-G. Meissner, Phys. Lett. B 198 (1987) 92; V. Bernard, R.L. Jaffe, U.-G. Meissner, Nucl. Phys. B 308 (1988) 753.

[11] H. Reinhardt, R. Alkofer, Phys. Lett. B 207 (1988) 482.

[12] S. Klimt, M. Lutz, U. Vogl, W. Weise, Nucl. Phys. A 516 (1990) 429; U. Vogl, M. Lutz, S. Klimt, W. Weise, Nucl. Phys. A 516 (1990) 469; U. Vogl, W. Weise, Progr. Part. Nucl. Phys. 27 (1991) 195.

[13] M. Takizawa, K. Tsushima, Y. Kohyama, K. Kubodera, Nucl. Phys. A 507 (1990) 611.

[14] S.P. Klevansky, Rev. Mod. Phys. 64 (1992) 649.

[15] T. Hatsuda, T. Kunihiro, Phys. Rep. 247 (1994) 221. Available from: <hep-ph/9401310>.

[16] V. Bernard, A.H. Blin, B. Hiller, U.-G. Meißner, M.C. Ruivo, Phys. Lett. B 305 (1993) 163. Available from: $<$ hep-ph/9302245>;

V. Dmitrašinović, Nucl. Phys. A 686 (2001) 379. Available from: <hep-ph/0010047>; K. Naito, M. Oka, M. Takizawa, T. Umekawa, Progr. Theor. Phys. 109 (2003) 969. Available from: $<$ hep$\mathrm{ph} / 0305078>$.

[17] A.A. Osipov, B. Hiller, V. Bernard, A.H. Blin, Ann. Phys. (NY) (in press), Available on line 18 April 2006. Available also from: $<$ hep-ph/0507226 $>$;

B. Hiller, A.A. Osipov, V. Bernard, A.H. Blin, SIGMA 2 (2006) 026. Available from: $<$ hep-ph/0602165 $>$.

[18] A.A. Osipov, B. Hiller, J. da Providência, Phys. Lett. B 634 (2006) 48. Available from: <hep-ph/ 0508058>.

[19] T. Eguchi, Phys. Rev. D 14 (1976) 2755.

[20] K. Kikkawa, Prog. Theor. Phys. 56 (1976) 947.

[21] D. Diakonov, Chiral symmetry breaking by instantons, Lectures at the Enrico Fermi school in Physics, Varenna, June 27-July 7, 1995. Available from: $<$ hep-ph/9602375>;

D. Diakonov, Chiral quark-soliton model, Lectures at the Advanced Summer School on Non-Perturbative Field Theory, Peniscola, Spain, June 2-6, 1997. Available from: <hep-ph/9802298>.

[22] J. Schwinger, Phys. Rev. 82 (1951) 664;

B.S. DeWitt, Dynamical Theory of Groups and Fields, Gordon and Breach, New York, 1965; B.S. DeWitt, Phys. Rep. 19 (1975) 295.

[23] R.D. Ball, Phys. Rep. 182 (1989) 1; D.V. Vassilevich, Phys. Rep. 388 (2003) 270. Available from: $<$ hep-th/0306138>.

[24] A.A. Osipov, B. Hiller, Phys. Lett. B 515 (2001) 458. Available from: $<$ hep-th/0104165>; A.A. Osipov, B. Hiller, Phys. Rev. D 63 (2001) 094009. Available from: <hep-ph/0012294>; A.A. Osipov, B. Hiller, Phys. Rev. D 64 (2001) 087701. Available from: <hep-th/0106226>.

[25] E. Witten, Nucl. Phys. B 160 (1979) 57. 
[26] S. Coleman, E. Witten, Phys. Rev. Lett. 45 (1980) 100;

C. Vafa, E. Witten, Nucl. Phys. B 234 (1984) 173.

[27] M.K. Volkov, Ann. Phys. (NY) 157 (1984) 282;

M.K. Volkov, Sov. J. Part. Nuclei 17 (1986) 186;

D. Ebert, H. Reinhardt, Nucl. Phys. B 271 (1986) 188;

J. Bijnens, C. Bruno, E. de Rafael, Nucl. Phys. B 390 (1993) 501. Available from: <hep-ph/ 9206236>.

[28] S. Okubo, Phys. Lett. B 5 (1963) 165;

G. Zweig, CERN Report No. 8419/TH412 (1964);

I. Iizuka, Progr. Theor. Phys. Suppl. 37-38 (1966) 21.

[29] A.A. Osipov, H. Hansen, B. Hiller, Nucl. Phys. A 745 (2004) 81. Available from: <hep-ph/0406112>.

[30] A.A. Osipov, B. Hiller, Phys. Lett. B 539 (2002) 76;

A.A. Osipov, B. Hiller, Eur. Phys. J.C 35 (2004) 223. Available from: <hep-th/0307035>.

[31] E. Witten, Ann. Phys. (NY) 128 (1980) 363;

R. Arnowitt, Pran Nath, Phys. Rev. D 23 (1981) 473;

Nucl. Phys. B 209 (1982) 234, 251.

[32] M. Creutz, Phys. Rev. D 52 (1995) 2951. Available from: <hep-th/9505112>;

I. Halperin, A. Zhitnitsky, Phys. Rev. Lett. 81 (1998) 4071. Available from: <hep-ph/9803301>;

A.V. Smilga, Phys. Rev. D 59 (1999) 114021. Available from: $<$ hep-ph/9805214>;

M. Shifman, Phys. Rev. D 59 (1999) 021501. Available from: <hep-th/9809184>;

M.H. Tytgat, Phys. Rev. D 61 (2000) 114009. Available from: <hep-ph/9909532>;

G. Gabadadze, M. Shifman, Int. J. Mod. Phys. A 17 (2002) 3689. Available from: <hep-ph/0206123>; P.J.A. Bicudo, J.E.F.T. Ribeiro, A.V. Nefediev, Phys. Rev. D 65 (2002) 085026. Available from: $<$ hep-ph/ 0201173>;

A.V. Nefediev, J.E.F.T. Ribeiro, Phys. Rev. D 70 (2004) 094020. Available from: <hep-ph/0409112>.

[33] P. Bicudo, Available from: <hep-ph/0606189>.

[34] A. Pais, Phys. Rev. 173 (1968) 1587.

[35] M. Gell-Mann, M. Lévy, Nuovo Cimento 16 (1960) 705.

[36] Particle Data Group, Review of Particle Properties, Phys. Lett. B 592 (2004) 1.

[37] R. Keiser, Diploma Work, University of Bern, 1997.

[38] H. Leutwyler, Proc. QCD 97, Montpellier, France, July 1997, in: S. Narison (Ed.), Nucl. Phys. B (Proc. Suppl.) 64 (1998) 223. Available from: $<$ hep-ph/9709408 $>$;

R. Keiser, H. Leutwyler, Available from: $<$ hep-ph/9806336>.

[39] T. Feldmann, P. Kroll, B. Stech, Phys. Rev. D58 (1998) 114006.

[40] T. Feldmann, Int. J. Mod. Phys. A15 (2000) 159. Available from: <hep-ph/9907491>.

[41] T. Feldmann, P. Kroll, B. Stech, Phys. Lett. B 449 (1999) 339.

[42] L. Del Debbio, L. Giusti, C. Pica, Phys. Rev. Lett. 94 (2005) 032003. Available from: <hep-th/0407052>.

[43] V. Dmitrašinović, Phys. Rev. C 53 (1996) 1383.

[44] J.D. Weinstein, N. Isgur, Phys. Rev. D 41 (1990) 2236;

J. Janssen, B.C. Pearce, K. Holinde, J. Speth, Phys. Rev. D 52 (1995) 2690;

M.P. Locher, V.E. Markushin, H.Q. Zheng, Eur. Phys. J.C 4 (1998) 317.

[45] N.N. Achasov, Nucl. Phys. A 728 (2003) 425.

[46] E. Klempt, Available from: <hep-ph/0409164>.

[47] E.M. Aitala et al., Phys. Rev. Lett. 89 (2002) 121801. Available from: <hep-ex/0204018>.

[48] C. Goebel, on behalf of the E791 Collaboration, Proceedings of Heavy Quarks at Fixed Target, Rio de Janeiro, October 2000, 373. Available from: $<$ hep-ex/0012009>.

[49] E. van Beveren, T.A. Rjken, K. Metzger, C. Dullemond, G. Rupp, J.E. Ribeiro, Z. Phys. C30 (1986) 615; E. van Beveren, G. Rupp, N. Petropoulos, F. Kleefeld, Effective Theories of Low Energy QCD, 2nd Int. Workshop on Hadron Physics, Coimbra, Portugal, AIP Conference Proceedings 660 (2003) 353.

[50] M. Napsuciale, S. Rodriguez, Int. J. Mod. Phys. A16 (2001) 3011. Available from: <hep-ph/ 0204149>;

M. Napsuciale, A. Wirzba, M. Kirchbach, Available from: <nucl-th/0105055>;

M. Napsuciale, Available from: <hep-ph/0204170>.

[51] M.K. Volkov, M. Nagy, V.L. Yudichev, Nuovo Chim. A 112 (1999) 225;

M.K. Volkov, V.L. Yudichev, Phys. Atom. Nucl. 62 (1999) 1567, [Yad. Fiz. 62 (1999) 1674]; 
M.K. Volkov, V.L. Yudichev, Eur. Phys. J.A 10 (2001) 109;

Y.-B. Dai, Y.-L. Wu, Eur. Phys. J.C 39 (2005) S1-S8. Available from: <hep-ph/0304075>.

[52] J.A. Oller, E. Oset, J.R. Peláez, Phys. ReV. D59 (1999) 074001 (J.A. Oller, E. Oset, J.R. Peláez, Phys. ReV.

D60 (1999) 099906). Available from: <hep-ph/9804209>.

[53] R.L. Jaffe, Phys. Rev. D 15 (1977) 267;

Yu. Kalashnikova, A. Kudryavtsev, A.V. Nefedief, J. Haidenbauer, C. Hanhart, Available from: $<$ hep-th/ 0512028>.

M. Uehara, Available from: $<$ hep-ph/0404221>.

[54] E. Klempt, B.C. Metsch, C.R. Münz, H.R. Petry, Phys. Lett. B 361 (1995) 160;

C. Ritter, B.C. Metsch, C.R. Münz, H.R. Petry, Phys. Lett. B 380 (1996) 431.

[55] A. Bramon, M.D. Scadron, Phys. Lett. B 234 (1990) 346;

A. Bramon, R. Escribano, M.D. Scadron, Eur. Phys. J.C 7 (1999) 271. Available from: $<$ hep-ph/9711229>.

[56] M. Jamin, Phys. Lett. B 538 (2002) 71.

[57] G.S. Bali, Phys. Rept. 343 (2001) 1. Available from: <hep-ph/0001312>. 